\title{
Non-esterified Very Long-chain Fatty Acids Are Accumulated in Colorectal Cancer Tissues
}

\section{Kotaro Hama ( $\nabla$ khama@pharm.teikyo-u.ac.jp )}

Teikyo University

\section{Yuko Fujiwara}

Teikyo University

\section{Tamuro Hayama}

Teikyo University

\section{Tsuyoshi Ozawa}

Teikyo University

Keijiro Nozawa

Teikyo University

Keiji Matsuda

Teikyo University

\section{Yojiro Hashiguchi}

Teikyo University

\section{Kazuaki Yokoyama}

Teikyo University

\section{Research Article}

Keywords: Fatty acid, Fatty acid/Metabolism, Lipids, Lipidomics, Very long-chain fatty acid (VLCFA), Nonesterified VLCFA, colorectal cancer (CRC), Elongation of very long-chain fatty acids 1 (ELOVL1), Fatty acid transport protein 4 (FATP4)

Posted Date: November 24th, 2020

DOl: https://doi.org/10.21203/rs.3.rs-109162/v1

License: (1) This work is licensed under a Creative Commons Attribution 4.0 International License. Read Full License

Version of Record: A version of this preprint was published at Scientific Reports on March 17th, 2021. See the published version at https://doi.org/10.1038/s41598-021-85603-w. 
Non-esterified very long-chain fatty acids are accumulated in colorectal cancer tissues

\title{
Kotaro Hama $^{1, *}$, Yuko Fujiwara ${ }^{1}$, Tamuro Hayama ${ }^{2}$, Tsuyoshi Ozawa ${ }^{2}$, Keijiro Nozawa ${ }^{2}$, Keiji Matsuda $^{2}$, Yojiro Hashiguchi ${ }^{2}$, and Kazuaki Yokoyama ${ }^{1}$
}

${ }^{1}$ Faculty of Pharma-Sciences, Teikyo University; ${ }^{2}$ Department of Surgery, Teikyo University School of Medicine, 2-11-1 Kaga, Itabashi-ku, Tokyo 173-8605, Japan

Running title: VLCFA in colorectal cancer tissues

*To whom correspondence and proofs should be addressed: Kotaro Hama, Faculty of PharmaSciences, Teikyo University, 2-11-1 Kaga, Itabashi-ku, Tokyo 173-8605, Japan; khama@pharm.teikyo-u.ac.jp; Tel: +81-3-3964-8197; Fax: +81-3-3964-8198

\begin{abstract}
Abbreviations: CRC, colorectal cancer; VLCFA, very long-chain fatty acid; ABCD1, ATPbinding cassette transporter subfamily D1; DB, double bond(s) between carbon-carbon atoms; ELOVL, elongation of very long-chain fatty acids; ACSL, acyl-CoA synthetase long-chain family member; FATP, fatty acid transport protein; IS, internal standard; LC, liquid chromatography; MS, mass spectrometry; PL, phospholipid
\end{abstract}




\begin{abstract}
Colorectal cancer (CRC) is a major cancer, and its precise diagnosis is especially important for the development of effective therapeutics. In a series of metabolome analyses, the levels of very long chain fatty acids (VLCFA) was shown to be elevated in CRC tissues, although the endogenous form of VLCFA has not been fully elucidated. In this study we analyzed the amount of non-esterified fatty acids, phospholipids and acyl-CoA species by liquid-chromatographymass spectrometry and showed that VLCFA is accumulated as the non-esterified form in CRC tissues. We also showed that the expression level of elongation of very long-chain fatty acids 1 (ELOVLI) is increased, whereas fatty acid transport protein 4 (FATP4) is decreased in CRC tissues. Finally, we showed that the amount of non-esterified VLCFA species was significantly up-regulated in cultured cells overexpressing ELOVL1. Our results suggest that the upregulation of ELOVL1 and the down-regulation of FATP4 cooperatively lead to the accumulation of non-esterified VLCFA in CRC tissues.
\end{abstract}

Keywords: Fatty acid, Fatty acid/Metabolism, Lipids, Lipidomics, Very long-chain fatty acid (VLCFA), Non-esterified VLCFA, colorectal cancer (CRC), Elongation of very long-chain fatty acids 1 (ELOVL1), Fatty acid transport protein 4 (FATP4)

\title{
Introduction
}

Globally, colorectal cancer (CRC) is the second and third highest cause of cancer in women and men, respectively, and is also the second most common cause of cancer related death in the United States and Japan [1]. A number of lifestyle factors are implicated as risk factors for CRC, and especially, a westernized diet with higher intake in total energy, saturated fat, sucrose and salt, and lower in fiber, is often described as the major risk factor for CRC. A recent cohort study reported that the intake of the $n-3$ polyunsaturated fatty acids (PUFA) reduced the risk of CRC 
occurrence, whereas the ratio of $n-3$ PUFA $/ n-6$ PUFA did not correlate with CRC occurrence [2], These results suggest that the pathological contribution of the amount of fatty acids and lipid metabolism in CRC remains to be elucidated.

Very long-chain fatty acids (VLCFA) with no less than 23 or 24 carbons are endogenously synthesized as very long-chain fatty acyl-CoA (VLCFA-CoA) through a fatty acid elongation process [3]. Then VLCFA-CoA are incorporated into complex lipids such as phospholipids (PL). Recently, several analysis using gas chromatography-mass spectrometry (GC-MS) revealed that VLCFA levels are elevated in plasma and surgical specimens from CRC patients [4]. However, the endogenous form of VLCFA in CRC tissues has not been fully understood due to the limited number of intensive lipidomic analyses focusing on VLCFA. Notably, fatty acyl moieties are liberated from complex lipids during the derivatizing process of sample preparation for GC-MS, which leads to loss of information about the endogenous lipid species containing VLCFA.

Advances in liquid chromatography-electrospray ionization-mass spectrometry (LC-ESIMS) have enabled simultaneous quantification of a large number of underivatized lipid species in biological samples. In this study, we analyzed underivatized lipid fractions from surgical specimens, from patients with CRC by LC-ESI-MS, and revealed that VLCFA are not accumulated as esterified, but as non-esterified form in CRC tissues. To gain a mechanistic insight, we also conducted expression analysis of genes involved in lipid metabolism in CRC tissues.

\section{Results}

\section{The level of VLCFA-CoA is not elevated in CRC tissue}

VLCFA are initially produced as VLCFA-CoA through de novo fatty acid elongation process [3]. Therefore, we first profiled the amount of VLCFA-CoA species in CRC tissues. Among 59 acylCoA species quantified in CRC and adjacent normal tissues, we found that $3 \%$ of the total acylCoA species consisted of the VLCFA-CoA species, with no less than 23 carbons in their fatty acyl moiety. Tetracosenoyl-CoA (24:1-CoA) was the most abundant VLCFA-CoA species in 
both CRC and adjacent normal tissues (Fig. 1A, and Supplementary Table S1 on line). We could not observe any VLCFA-CoA species that significantly accumulated in CRC tissues (Fig. 1A), but rather, we found that the amount of two VLCFA-CoA species (23:0-CoA and 24:0-CoA) was significantly smaller in CRC tissues, than in normal tissues (Fig. 1B). These results show that the intracellular pool of VLCFA-CoA is not significantly increased in CRC tissues.

\section{The level of phospholipid species containing VLCFA is not altered in CRC tissues}

Acyl-CoA species serve as metabolic intermediates of fatty acids involved in the synthesis of complex lipids, post-translational modification and $\beta$-oxidation. PLs that mostly contain two acylmoieties are abundantly present as membrane components in cells, and thus could serve as an intracellular reservoir for free fatty acids. Therefore, we next analyzed PL species to examine whether VLCFA species are accumulated in esterified form in CRC tissues. We analyzed 39 PL species (7 PC, 7 PE, 7 PS, 7 PI, 7 PG and 4 SM species), in which acyl moieties were determined by scanning product ions from parental ions of each PL species. The amounts of most PL species (17 out of 19 species) with long-chain fatty acyl moiety (C16:0, C18:0, C18:1, C20:4 and C22:0) were significantly lower in CRC tissues than in adjacent normal tissues, and the amounts of only 7 out of 20 PL species with VLCFA moiety (C24:0 and C26:0) were smaller in CRC tissue (Fig. 2A, B and Supplementary figure S1 online). We could not observe PL species, which amount was significantly higher in CRC tissues. These results show that VLCFA are not accumulated as esterified form in PL species, and indicate that the PL metabolism could be different according to the length of fatty acyl moieties of PL species in CRC tissues.

\section{The level of non-esterified VLCFA is elevated in CRC tissues}

Non-esterified VLCFA could be produced through deactivating VLCFA-CoA by acyl-CoA thioesterases or liberation from complex lipids such as PL by lipases. To profile the FFA species as non-esterified fatty acids in CRC tissues, we analyzed the underivatized FFA in total lipid 
fractions by LC-MS method. We used a $\mathrm{C}_{8}$ reversed-phase column and mobile phases adjusted to pH 9.0 by ammonium hydroxide, which are almost identical with the methods for acyl-CoA analyses developed so far [5]. We validated our method using two isotopically-labeled palmitic acids, and confirmed that 8-2000 pmol/injection of FFA species can be quantified with the present quantitative method (Supplementary Table S2 online). We then profiled each FFA species in CRC tissues from 24 patients. The amount of FFA 16:0 in CRC was significantly lower than in adjacent normal tissues. By contrast, the amount of non-esterified VLCFA species (FFA 24:0, 24:1, 26:0, and FFA 26:1) and FFA 22:1 was significantly higher in CRC tissues than in normal tissues (Fig. 3A). The portion of four non-esterified VLCFA species with no less than 24 carbons (FFA 24:0, 24:1, 26:0, 26:1) was also significantly higher in CRC than in adjacent normal tissues (Fig. 3B). These results show that VLCFA species are accumulated as non-esterified form in CRC tissues.

The expression level of ELOVL1 is correlated with non-esterified VLCFA level in CRC tissue

To explore the machinery that explains how non-esterified VLCFA is accumulated in CRC tissues, we first focused on the process of fatty acyl elongation. ELOVL1 and ABCD1 (ATP-binding cassette transporter subfamily D1) play critical roles in the de novo synthesis and degradation of VLCFA, respectively $[3,6]$. We examined the mRNA levels of ELOVL1 and $A B C D 1$ in samples from 40 CRC patients, and found that the expression level of ELOVL1 was higher in CRC tissues than in adjacent normal tissues (Fig 4A), a result which is consistent with a previous report [7]. In contrast, the expression level of $A B C D 1$ was not significantly altered (Fig 4A). We also tested the significance of correlation by Pearson's correlation coefficient between each variable, consisting of CRC/normal ratio of ELOVL1 mRNA and the 15 FFA species analyzed in this study. We found that the CRC/normal ratio of ELOVL1 mRNA is positively and significantly correlated with the CRC/normal ratio of saturated (FFA 20:0 and FFA 22:0) or mono-unsaturated FFA (FFA 
22:1, FFA 24:1 and FFA 26:1) (Fig. 4B). These results suggest that ELOVL1 is involved in the accumulation of saturated or mono-unsaturated non-esterified fatty acids with no less than 20 carbons in CRC tissues.

\section{The expression level of FATP4 is down-regulated in CRC tissues}

Our lipidomic study revealed that the expression level of ELOVLI is significantly increased, while VLCFA is accumulated as non-esterified form, but not as VLCFA-CoA or VLCFA-PL in CRC tissues (Fig. 1-3). This discrepancy led us to examine the re-activation process of VLCFA by acyl-CoA synthetases, which convert non-esterified fatty acids into acyl-CoA species. Long chain fatty acyl-CoA synthetases (ACSL), long chain fatty acid transport proteins (FATP) and bubblegum (ACSBG) are especially important for the activation and metabolism of long chain fatty acids [8]. We thus quantified the mRNA levels of ACSL (ACSL1, and 3-6), FATP (FATP16) and $A C S B G$ (ASCBG1 and 2), and found that only the expression level of FATP4 was significantly decreased in CRC tissues, as compared with adjacent normal tissues (Fig. 5). We further examined another possibility that non-esterified VLCFA is accumulated due to increased rate of hydrolysis of ceramides by ceramidases, since the amount of SM species was grossly decreased in CRC tissues (Fig. 2). We analyzed two acid ceramidases (ASAH1 and 2) and three neutral ceramidases (ACER1, 2 and 3), although a significant change could not be observed in the amount of these five ceramidases (Fig. 5). FATP4 is the gene responsible for ichthyosis prematurity syndrome, and it has been shown that FATP4 prefers very long-chain free fatty acids with no less than 24 carbons as substrate [9-11]. Therefore, these results indicate that the accumulation of non-esterified VLCFA is caused by the combined effects of up-regulation of ELOVL1 and down-regulation of FATP4 in CRC tissues.

ELOVL1 contributes to the accumulation of non-esterified and esterified VLCFA in cultured cells 
Finally, we confirmed the role of ELOVL1 in the accumulation of non-esterified VLCFA. The amount of saturated and mono-unsaturated non-esterified VLCFA species (FFA 24:0, FFA24:1, FFA26:0 and FFA 26:1) were significantly increased in HEK293T cells transiently transfected with ELOVL1 (Fig. 6A). Importantly, the amount of both four VLCFA-CoA (24:0-, 24:1:-, 26:1and 28:1-CoA) and 10 PL species (3 PC, 1 PE, 4 PS and 2 PI species) harboring C24:0 or C26:0 as their acyl moiety were also significantly increased in HEK293T cells transiently transfected with ELOVL1 (Fig. 6B-D and Supplementary Table S3 online). Notably, the amount of 26:0-CoA was much smaller than that of 26:1-CoA, whereas the amount of FFA 26:0 was relatively close to that of FFA 26:1 in both mock and ELOVL1 transfected cells (Fig. 6A and B). These results suggest that saturated VLCFA-CoA synthesized by ELOVL1 is efficiently metabolized in CRC tissues.

\section{Discussion}

In this study, we found that saturated and mono-unsaturated VLCFA are accumulated as non-esterified form but not as esterified form such as acyl-CoA or phospholipid species, in CRC tissues (Fig. 1-3). This result is partially consistent with a previous report, in which FFA 24:0 and FFA 24:1 were shown to be accumulated in both CRC tissues with and without lymph node metastasis [12]. It will be necessary to examine whether esterified VLCFA is accumulated in other lipid species such as glycolipids and neutral lipids, including triacylglycerol and cholesterylester, in CRC tissues. It has also been shown that the amount of total VLCFA, including esterified and non-esterified forms, are elevated in the sera of colorectal cancer patients [13]. It needs to be further examined whether the accumulation of non-esterified VLCFA in CRC tissues directly leads to the changes in the composition of serum fatty acids by comparing the fatty acid composition in the sera of pre- and post-surgery patients.

It is still unclear that how FATP4 is involved in the cancer progression in CRC tissues through the regulation of VLCFA metabolism. Recent analysis showed that the expression level 
of FATP4 in cancer tissues is inversely correlated with survival rates in breast cancer [14]. In contrast, we showed that the expression level of FATP4 is decreased in CRC tissues (Fig. 5), suggesting that FATP4 expression could be different between different types of cancer. Further analysis should be undertaken to clarify So far, we inhibited FATP4 expression by siRNA in ABCD1-deficient HeLa cells, in which PL species and acyl-CoA species harboring VLCFA moiety are accumulated [5]. However, significant difference could not be observed in the amount of non-esterified VLCFA, as compared with control-siRNA treated cells (data not shown). Considering the higher expression level of ELOVL1 in CRC tissues (Fig. 4A), our results suggest that FATP4 may contribute to the accumulation of non-esterified VLCFA in the condition of excessive ELOVL1 expression.

It is possible that several lipid metabolic pathways are involved in the accumulation of nonesterified VLCFA in CRC tissues. Firstly, acyl-CoA thioesterase could catalyze the hydrolysis of VLCFA-CoA to non-esterified VLCFA and CoA. A number of acyl-CoA thioesterases have been identified so far and implicated in lipid metabolism, such as peroxisomal $\beta$-oxidation [15]. Thus, some acyl-CoA thioesterases that prefer VLCFA-CoA as a substrate, are activated and contribute to the accumulation of non-esterified VLCFA. Secondly, non-esterified VLCFA is released from complex lipids such as phospholipids. Especially, we observed significant decline in the amount of glycerophospholipids with VLCFA such as PC 24:0/16:0, PC 26:0/16:0, PE 24:0/16:0, PS 24:0/16:0, PS 24:0/18:1, PG 24:0/18:1 and PG 26:0/18:1 (Fig. 2 and Supplementary figure S1, online). It has been shown that the VLCFA moiety is located at the $s n-1$ position of the glycerol backbone of PL [5,16-18]; thus it is also possible that phospholipase $A_{1}$ contributes to the accumulation of non-esterified VLCFA in CRC tissues. Three intracellular phospholipase $\mathrm{A}_{1}$ identified so far in mammals (PA-PLA1 $\alpha /$ DDHD1/iPLA1 $\alpha$, p125/Se23IP/iPLA1 $\beta$ and KIAA0725p/DDHD2/iPLA1 $\gamma$ ) prefer PA, PE and PI but not PC as substrates [19]. Therefore, intracellular phospholipase $\mathrm{A}_{1}$ and phospholipase $\mathrm{D}$ cooperatively contribute to the production of non-esterified VLCFA. Note that ELOVL1 overexpression in HEK293T cells resulted in not only 
the accumulation of non-esterified VLCFA, but also esterified VLCFA such as acyl-CoA and PL (Fig. 6A-D). In contrast, VLCFA is accumulated as non-esterified but not as esterified form in CRC tissues (Fig. 1-3). These differences in the VLCFA profile might be attributed to the difference in the activity of enzymes involved in VLCFA metabolism such as acyl-CoA thieoesterases and intracellular phospholipase $\mathrm{A}_{1}$, between CRC tissues and HEK293T cells.

The present study showed that the amount of SM species with VLCFA was significantly decreased in CRC tissues (Fig. 2). A recent study showed that SM species containing VLCFA and cholesterol cooperatively play crucial roles in maintaining tight junctions, which is essential for the barrier functions of enterocytes [20]. VLCFA-CoA is transferred into sphingosine by ceramide synthases, and then ceramide species with VLCFA are converted into SM by sphingomyelin synthases. Therefore, it might be possible that the down-regulation of FATP4 expression causes the decline in the amount of VLCFA-CoA and VLCFA-containing SM, which leads to aberrant tight junctions in colonic epithelial cells.

\section{Materials and Methods}

\section{Ethics}

This study abides by the Declarations of Helsinki Principles, and the research protocol was approved by the Ethics Committee of Teikyo University (\#19-153). We enrolled 40 consecutive patients treated with curative resection at Teikyo University Hospital, Japan during the period 2019-2020. The surgery of all of the patients was elective. Informed consent was obtained from all participants and the reporting of our research is in accordance with the STROBE guidelines [21].

\section{Reagents}

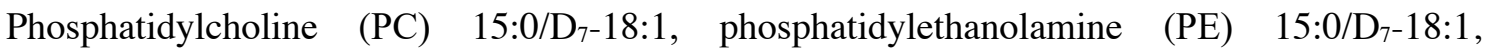
phosphatidylserine (PS) 15:0/D $\mathrm{D}_{7}-18: 1, \quad$ phosphatidylinositol $\quad$ (PI) 15:0/D $-18: 1$, 


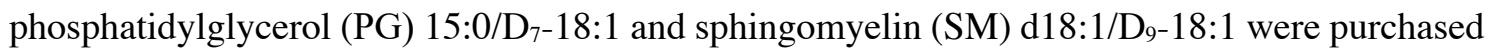
from Avanti Polar Lipids, Inc. (Alabaster, AL, USA). All chemicals used in the mobile phases were purchased from Fujifilm Wako Pure Chemical Corporation (Osaka, Japan). Deuteriumlabeled $\left(7,7,8,8-\mathrm{D}_{4}\right)$ palmitic acid $\left(\mathrm{FA} \mathrm{D}_{4}-16: 0\right)$ and $\mathrm{FA} \mathrm{D}_{31}-16: 0$ were purchased from Cambridge Isotope Laboratories, Inc. (Andover, MA, USA), and Santa Cruz Biotechnology (Dallas, TX, USA), respectively.

\section{Sample preparation}

All colorectal tissue specimen were dissected into small pieces, $\sim 5 \mathrm{~mm}$. a side, frozen in liquid nitrogen within 24 hours after surgery, and stored at $-80^{\circ} \mathrm{C}$ until lipid extraction. Sample preparation for PL and free fatty acid (FFA) analysis was conducted as reported previously with slight modification $[18,22]$. Briefly, the tissue specimen were homogenized in $1 \mathrm{~mL}$ of methanol using a Micro Smash MS-100R instrument (TOMY, Tokyo, Japan) at $3000 \mathrm{rpm}$ for $10 \mathrm{~s}$ at $4^{\circ} \mathrm{C}$. This procedure was repeated 3 to 6 times until the tissues were mostly homogenized. Then, an

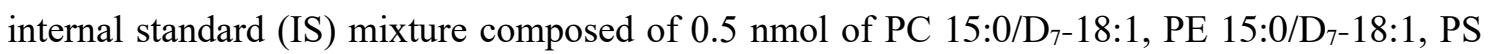

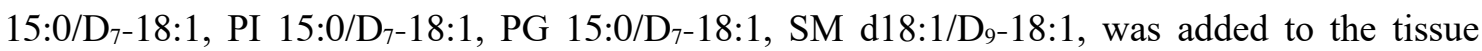
homogenate, and the total lipid fraction was extracted by the Bligh and Dyer method [23]. The resulting lower organic phase was evaporated with the EZ-2 centrifugal evaporator (Genevac, Ipswich, UK), and the resulting precipitate was reconstituted with $0.1 \mathrm{~mL}$ of ethanol, followed by filtration with a YMC Duo-Filter (4 mm i.d., pore size $0.2 \mu \mathrm{m}$; YMC Co., Ltd., Kyoto, Japan). Samples were stored at $-20^{\circ} \mathrm{C}$ until further analysis. For acyl-CoA analysis, the tissue specimen were homogenized in $0.9 \mathrm{~mL}$ of acetonitrile/isopropanol (3:1 by volume) and the acyl-CoA fraction was extracted as previously described [5]. Samples were stored at $-20^{\circ} \mathrm{C}$ until further analysis. Homogenate protein concentrations were determined with a BCA protein assay kit (Thermo Fisher Scientific, Waltham, MA). The samples for the validation of the LC-MS method for FFA were prepared as described in supplemental materials online. 


\section{LC-MS/MS analysis}

Quantitation of each PL species was conducted with a TSQ Quantum Ultra (Thermo Fisher Scientific) linked to an Accela HPLC system (Thermo Fisher Scientific). An InertSustain $\mathrm{C}_{18}$ metal-free column (2.1 mm i.d. $\times 50 \mathrm{~mm}$, particle size $3.0 \mu \mathrm{m}$; GL Sciences, Tokyo, Japan) was used at $50^{\circ} \mathrm{C}$. The mobile phases were acetonitrile/methanol/water $(2: 2: 1$, by vol.) with $0.1 \%$ formic acid and $0.028 \%$ ammonia (A), and isopropanol with $0.1 \%$ formic acid and $0.028 \%$ ammonia (B). The programmed solvent gradient consisted of solvents $\mathrm{A} / \mathrm{B}$ at $70: 30$ ratio for 1 min, linear alteration to 5:95 over $10 \mathrm{~min}$, hold at 5:95 over $4 \mathrm{~min}$, linear conversion to 70:30 over $1 \mathrm{~min}$ and then hold at 70:30 over $2.5 \mathrm{~min}$. The flow rate was $280 \mu \mathrm{L} / \mathrm{min}$, and the volume of the injected samples was $5 \mu \mathrm{L}$. The analysis was conducted in the positive ion mode. The time period for data collection was $25 \mathrm{~ms} /$ cycle for each multiple reaction monitoring (MRM) transition. The following conditions were used for positive ion MRM: ion spray voltage, $3.5 \mathrm{kV}$; vaporizer temperature, $287^{\circ} \mathrm{C}$; sheath gas pressure, $10 \mathrm{psi}$; auxiliary gas pressure, $35 \mathrm{psi}$; collision gas pressure, $0.7 \mathrm{mTorr}$; capillary temperature, $320^{\circ} \mathrm{C}$; tube lens offset $133 \mathrm{~V}$. Nitrogen was used as sheath and auxiliary gas, whereas argon was used as collision gas. Xcalibur ${ }^{\mathrm{TM}}$ software (Thermo Fisher Scientific) was used for data acquisition and processing. The fatty acyl moieties in each PL species were determined by structural analysis in negative ion mode conducted with a QTRAP 4500 (Sciex, Framingham, MA, USA) linked to a Nexera XR HPLC system (Shimadzu Corp., Kyoto, Japan) as described previously [18]. The fatty acyl moieties of each PL were assigned by the detection of the product ions from fatty acyl residues and lysophospholipids. In addition, fatty acyl residue positions were also determined by comparing the spectral intensity of two lysophospholipid ions produced from precursor ions, as reported previously [24]. Quantitation of each FFA species was conducted with a TSQ Quantum Ultra linked to an Accela HPLC system. A Capcell Pak C 8 UG120 column $(1.5 \mathrm{~mm}$ i.d. $\times 35 \mathrm{~mm}$, particle size $5.0 \mu \mathrm{m}$; Shiseido Co., Ltd., Tokyo, Japan) was used at $40^{\circ} \mathrm{C}$. The mobile phases were $5 \mathrm{mM}$ ammonium 
formate in water (pH 9.0) (A) and $5 \mathrm{mM}$ ammonium formate in a water/isopropanol solution (5:95 by volume; $\mathrm{pH}$ 9.0) (B). The programmed solvent gradient consisted of solvents $\mathrm{A} / \mathrm{B}$ at $70: 30$ ratio for $1 \mathrm{~min}$, linear alteration to 5:95 over $10 \mathrm{~min}$, hold at 5:95 over $4 \mathrm{~min}$, linear conversion to $70: 30$ over $1 \mathrm{~min}$ and then hold at 70:30 over $2.5 \mathrm{~min}$. The flow rate was $200 \mu \mathrm{L} / \mathrm{min}$, and the volume of the injected samples was $10 \mu \mathrm{L}$. The analysis was conducted in the negative ion mode. The time period for data collection was $40 \mathrm{~ms} /$ cycle for each MRM transition. The following conditions were used for negative ion MRM: ion spray voltage, $2.5 \mathrm{kV}$; vaporizer temperature, $0^{\circ} \mathrm{C}$; sheath gas pressure, $10 \mathrm{psi}$; auxiliary gas pressure, $35 \mathrm{psi}$; collision gas pressure, $0.7 \mathrm{mTorr}$; capillary temperature, $270^{\circ} \mathrm{C}$; tube lens offset $146 \mathrm{~V}$. Nitrogen was used as sheath and auxiliary gas, whereas argon was used as collision gas. Each MRM transition for PL and FFA species used in the LC-MS/MS analysis is listed in Supplementary Table S4 online. Xcalibur ${ }^{\mathrm{TM}}$ software (Thermo Fisher Scientific) was used for data acquisition and processing. Quantitation of each acyl-CoA species was conducted with a QTRAP 4500 (Sciex, Framingham, MA, USA) linked to a Nexera XR HPLC system (Shimadzu Corp., Kyoto, Japan) as described previously (Hama K et al., 2020).

\section{Quantitative Real-time RT-PCR}

Total RNAs from colorectal tissues were extracted using the ISOGEN kit (Nippongene, Toyama, Japan) and cDNA libraries were synthesized with a high capacity cDNA RT kit (Thermo Fisher Scientific). The sequences of the oligonucleotides used in the PCR reaction are listed in Supplementary Table S5 online.

\section{Elongation of very long-chain fatty acids 1 (ELOVL1) overexpression in cultured cells}

HEK293T cells (Riken Cell Bank, Riken Bioresource Center, Ibaraki, Japan) were cultured in DMEM (Sigma-Aldrich, Inc., St. Louis, MO, USA) supplemented with 10\% fetal bovine serum, $2 \mathrm{mM} L$-glutamine (Thermo Fisher Scientific Inc., Waltham, MA, USA), $100 \mathrm{U} \mathrm{ml}^{-1}$ penicillin, 
$100 \mu \mathrm{g} \mathrm{ml}^{-1}$ streptomycin (Sigma-Aldrich, Inc.) and 1\% non-essential amino acids (Fujifilm Wako Pure Chemical Corporation). Cells were transiently transfected with the pcDNA3.1-human ELOVL1 (elongation of very long-chain fatty acids 1) plasmid using Lipofectamine 2000 reagent (Thermo Fisher Scientific, Inc.), according to the manufacturer's instructions. The pcDNA3.1human ELOVL1 plasmid was constructed as described previously [25]. Three days after transfection, cell layers were washed with phosphate buffered saline, scraped from the dishes, homogenized in methanol, and used for FFA and acyl-CoA extraction as described above. Homogenate protein concentrations were determined using a BCA protein assay kit (Thermo Fisher Scientific, Inc.).

\section{Statistical Methods}

Statistical analysis was performed with either Student's two-tailed $t$-test, two-tailed paired $t$-test or Pearson correlation coefficient. Differences were considered to be significant if the $p$-value was $<0.05$. All statistical analyses were conducted with IBM SPSS Statistics version 27 (IBM, Armonk, NY, USA).

\section{Data availability statement}

The datasets obtained in the current study are available from the corresponding author on reasonable request.

Acknowledgments The authors thank T. Yamanobe, K. Kurosaki, and colleagues for their technical assistance with mass spectrometry. This work was supported by a grant from the Science Research Promotion Fund of the Promotion and Mutual Aid Corporation for Private Schools of Japan (K.H.). This work was also supported by research grants from The Fugaku Trust for Medical Research (K.H.), and Advanced Comprehensive Research Grants of Teikyo University (K.H.). 
Contributions K.H. designed the study, performed the research and analyzed the data; K.H., Y.F. and K.Y. wrote the manuscript; T.H., T.O., K.N., K.M. and Y.H. contributed for acquisition of clinical samples and data. All authors reviewed the manuscript.

Conflict of Interest The authors declare that they have no conflict of interest. 


\section{References}

[1] Bray, F., Ferlay, J., Soerjomataram, I., Siegel, R.L., Torre, L.A. \& Jemal, A. Global cancer statistics 2018: GLOBOCAN estimates of incidence and mortality worldwide for 36 cancers in 185 countries. CA Cancer J. Clin. 68, 394-424 (2018).

[2] Sasazuki, S., Inoue, M., Iwasaki, M., Sawada, N., Shimazu, T., Yamaji, T., Takachi, R. \& Tsugane S. Intake of $n-3$ and $n-6$ polyunsaturated fatty acids and development of colorectal cancer by subsite: Japan Public Health Center-based prospective study. Int. J. Cancer 129, 1718-1729 (2011).

[3] Ofman, R., Dijkstra, I.M., van Roermund, C.W., Burger, N., Turkenburg, M., van Cruchten, A., van Engen, C.E., Wanders, R.J. and Kemp, S. The role of ELOVL1 in very long-chain fatty acid homeostasis and X-linked adrenoleukodystrophy. EMBO Mol. Med. 2, 90-97 (2010).

[4] Pakiet, A., Kobiela, J., Stepnowski, P., Sledzinski, T. and Mika A. Changes in lipids composition and metabolism in colorectal cancer: a review, Lipids Health Dis. 18, 29. 10.1186/s12944-019-0977-8 (2019).

[5] Hama, K., Fujiwara, Y., Takashima, S., Hayashi, Y., Yamashita, A., Shimozawa, N. and Yokoyama K. Hexacosenoyl-CoA is the most abundant very long-chain acyl-CoA in ATP binding cassette transporter D1-deficient cells. J. Lipid Res. 61, 523-536 (2020).

[6] Tsuji, S., Ohno, T., Miyatake, T., Suzuki, A. and Yamakawa T. Fatty acid elongation activity in fibroblasts from patients with adrenoleukodystrophy (ALD). J Biochem 96, 1241-1247 (1984).

[7] Mika A., Kobiela, J., Czumaj, A., Chmielewski, M., Stepnowski, P. and Sledzinski, T. Hyper-Elongation in Colorectal Cancer Tissue - Cerotic Acid is a Potential Novel Serum Metabolic Marker of Colorectal Malignancies. Cell Physiol. Biochem. 41, 722-730 (2017).

[8] Watkins P.A. Very-long-chain acyl-CoA synthetases. J. Biol. Chem. 283, 1773-1777 (2008).

[9] Hall, A.M., Wiczer, B.M., Herrmann, T., Stremmel, W. and Bernlohr, D.A. Enzymatic properties of purified murine fatty acid transport protein 4 and analysis of acyl-CoA synthetase activities in tissues from FATP4 null mice, J. Biol. Chem. 280, 11948-11954 (2005).

[10] Yamamoto, H., Hattori, M., Chamulitrat, W., Ohno, Y. and Kihara, A. Skin permeability barrier formation by the ichthyosis-causative gene FATP4 through formation of the barrier lipid $\omega$-O-acylceramide. Proc. Natl. Acad. Sci. U.S.A. 117, 2914-2922 (2020).

[11] Klar, J., Schweiger, M., Zimmerman, R., Zechner, R., Li, H., Törmä, H., Vahlquist, A., Bouadjar, B., Dahl, N. and Fischer, J. Mutations in the fatty acid transport protein 4 gene cause the ichthyosis prematurity syndrome. Am. J. Hum. Genet. 85, 248-253 (2009). [12] Chen, L., Chen, H., Li, Y., Li, L., Qiu, Y. and Ren J. Endocannabinoid and ceramide levels are altered in patients with colorectal cancer. Oncol. Rep. 34, 447-454 (2015).

[13] Kondo, Y., Nishiumi, S., Shinohara, M., Hatano, N., Ikeda, A., Yoshie, T., Kobayashi, T., Shiomi, Y., Irino, Y., Takenawa, T., Azuma, T. and Yoshida M. Serum fatty acid profiling of colorectal cancer by gas chromatography/mass spectrometry. Biomark. Med 5 451-460 (2011).

[14] Yen, M.C., Chou, S.K., Kan, J.Y., Kuo, P.L., Hou, M.F. and Hsu Y.L. Solute Carrier Family 27 Member 4 (SLC27A4) Enhances Cell Growth, Migration, and Invasion in Breast Cancer Cells. Int. J. Mol. Sci. 19, 10.3390/ijms 19113434 (2018). 
[15] Hunt, M.C. Siponen, M.I. and Alexson, S.E. The emerging role of acyl-CoA thioesterases and acyltransferases in regulating peroxisomal lipid metabolism. Biochim. Biophys. Acta. 1822, 1397-1410 (2012).

[16] Yokoyama, K., Saitoh, S., Ishida, M., Yamakawa, Y., Nakamura, K., Inoue, K. Taguchi, R., Tokumura, A., Nishijima, M., Yanagida, M. and Setaka M. Very-long-chain fatty acid-containing phospholipids accumulate in fatty acid synthase temperaturesensitive mutant strains of the fission yeast Schizosaccharomyces pombe fas $2 / l$ sd 1 . Biochim. Biophys. Acta. 1532, 223-233 (2001).

[17] Hama, K., Nagai, T., Nishizawa, C., Ikeda, K., Morita, M., Satoh, N., Nakanishi, H., Imanaka, T., Shimozawa, N., Taguchi, R., Inoue, K. and Yokoyama K. Molecular species of phospholipids with very long chain fatty acids in skin fibroblasts of Zellweger syndrome. Lipids 48, 1253-1267 (2013).

[18] Hama, K., Fujiwara, Y., Morita, M., Yamazaki, F., Nakashima, Y., Takei, S., Takashima, S., Setou, M., Shimozawa, N., Imanaka, T. and Yokoyama K. Profiling and Imaging of Phospholipids in Brains of Abcd1-Deficient Mice. Lipids 53, 85-102 (2018). [19] Tani K., Kogure T. and Inoue H. The intracellular phospholipase A1 protein family. Biomol. Concepts 3, 471-478 (2012).

[20] Shigetomi, K. Ono, Y. Inai, T. and Ikenouchi, J. Adherens junctions influence tight junction formation via changes in membrane lipid composition. J. Cell Biol. 217, 23732381 (2018).

[21] von Elm, E., Altman, D.G., Egger, M., Pocock, S.J., Gøtzsche, P.C. and Vandenbroucke, J.P. The Strengthening the Reporting of Observational Studies in Epidemiology (STROBE) Statement: guidelines for reporting observational studies. Int. J. Surg. 12, 1495-1499 (2014).

[22] Hama, K., Fujiwara, Y. and Yokoyama, K. Quantitative and Qualitative Method for Sphingomyelin by LC-MS Using Two Stable Isotopically Labeled Sphingomyelin Species. J. Vis. Exp. 10.3791/57293 (2018).

[23] Bligh, E.G. and Dyer, W.J. A rapid method of total lipid extraction and purification, Can. J. Biochem. Physiol. 37, 911-917 (1959).

[24] Nakanishi, H., Iida, Y., Shimizu, T. and Taguchi R. Separation and quantification of $s n-1$ and $s n-2$ fatty acid positional isomers in phosphatidylcholine by RPLC-ESIMS/MS. $J$ Biochem 147, 245-256 (2010).

[25] Hama, K., Fujiwara, Y., Tabata, H., Takahashi, H. and Yokoyama K. Comprehensive Quantitation Using Two Stable Isotopically Labeled Species and Direct Detection of $\mathrm{N}$ Acyl Moiety of Sphingomyelin. Lipids 52, 789-799 (2017). 
Fig. 1 The amount of acyl-CoA species in CRC tissues

(A) Each acyl-CoA species in adjunct normal tissues $(n=15)$ was quantified, and was classified according to the number of carbon and double bonds in the acyl moiety. The ratio of peak area for each acyl-CoA/D ${ }_{31}-16: 0-C o A$ was used to calculate the amount of each acyl-CoA species and the mean quantity of each acyl-CoA species in the tissues is represented with a color key. The data are also summarized in Supplymentary Table S1 online. Acyl-CoA species observed to be present below the quantitation range are indicated in gray. The total amount of the top three acylCoA species (16:0-, 18:0-, 18:1-CoA) comprises more than $50 \%$ of the total amount of all acylCoA species, analyzed within quantitative range. (B) The amount of 23:0- and 24:0-CoA species in CRC tissues and adjunct normal tissues. The amount of 23:0- and 24:0-CoA species in CRC tissues was significantly smaller than in adjunct normal tissues. Data represent the mean $\pm \mathrm{SD}$. Statistical analysis was performed with two-tailed paired $t$-test in (B).

Fig. 2 Quantity of each phospholipid (PL) species in the CRC tissues

(A) The amount of each PL species was quantified by LC-MS in positive ion mode. The lipid species present in quantities significantly lower or higher in CRC tissues $(n=24)$ relative to the adjunct normal tissues $(n=24)$ is presented in lime and magenta respectively. PL species for which the multiple reaction monitoring (MRM) channels were not designed are indicated in dot box. PL species with VLCFA moiety (C24:0 and C26:0) are indicated in blue dashed line. The amount of each phosphatidylcholine (PC) (B) and sphingomyelin (SM) species (C) are represented. The acyl-moieties for each PL species were determined by LC-MS/MS/MS in negative ion mode. Data represent the mean $\pm \mathrm{SD}$. Statistical analysis was performed with twotailed paired $t$-test in (A and B).

Fig. 3 Quantity of each non-esterified fatty acid (free fatty acid, FFA) species in the colorectal cancer (CRC) tissues 
Each FFA species (A) and the portion of non-esterified VLCFA (B) was analyzed by LC-MS. All of the non-esterified VLCFA consisting of FFA 24:0, 24:1, 26:0 and 26:1 were accumulated in CRC tissues. Data represent the mean $\pm \mathrm{SD}$. Statistical analysis was performed with two-tailed paired $t$-test in (A and $\mathrm{B})$.

Fig. 4 The expression level of ELOVL1 is correlated with saturated and mono-unsaturated nonesterified fatty acids with no less than 20 carbons

(A) The mRNA level of ELOVL1 and ABCD1 in CRC tissues and adjunct normal tissues. The expression level of ELOVL1 was significantly higher in CRC tissues. Data represent the mean \pm SD. (B) The correlation between the CRC/normal ratio of ELOVL1 mRNA and FFA. The higher ratio of ELOVL1 mRNA was positively correlated with the ratio of saturated and monounsaturated non-esterified fatty acids with no less than 20 carbons. Statistical analysis was performed with two-tailed paired $t$-test in (A) and Pearson correlation coefficient in (B).

Fig. 5 The expression level of acyl-CoA synthetases and ceramidases in CRC tissues The mRNA level of 13 acyl-CoA synthetases (ACSL1, 3-6, FATP1-6, and ACSBG1 and 2) and 5 ceramidases ( $A S A H 1$ and 2, and $A C E R 1-3)$ in CRC and adjunct normal tissues was quantified by quantitative PCR analysis. The expression level of FATP4 was significantly lower in CRC tissues. Data represent the mean $\pm \mathrm{SD}$. Statistical analysis was performed with two-tailed paired $t$-test.

Fig. 6 The amount of FFA (A), acyl-CoA (B) and PL (C, D) in HEK293T cells transfected with pcDNA3.1-human ELOVL1. (C) The lipid species present in quantities significantly higher in ELOVL1 overexpressing cells than in mock transfected cells are presented in magenta. PL species for which the multiple reaction monitoring (MRM) channels were not designed are indicated in dot box. PL species with VLCFA moiety (C24:0 and C26:0) are indicated in blue dashed line. (D) The amount of each PL species with VLCFA moiety are represented. The acyl-moieties for each 
PL species were determined by LC-MS/MS/MS in negative ion mode. Significant changes were not observed in the amount of PG species with VLCFA moiety. Note that both non-esterified (FFA) and esterified VLCFA (acyl-CoA and PL) were significantly accumulated in HEK293T cells transfected with ELOVL1. Data represent the mean \pm SD. Statistical analysis was performed with Student's two-tailed $t$-test in (A-D).

Fig. S1 Quantity of each phospholipid (PL) species in the CRC tissues The amount of each PE, PS, PI and PG species were quantified by LC-MS in positive ion mode. The acyl-moieties for each PL species were determined by LC-MS/MS/MS in negative ion mode. Data represent the mean $\pm \mathrm{SD}$. Statistical analysis was performed with two-tailed paired $t$-test. ${ }^{*} p$ $<0.05, * * p<0.01$ and $* * * p<0.001$ 

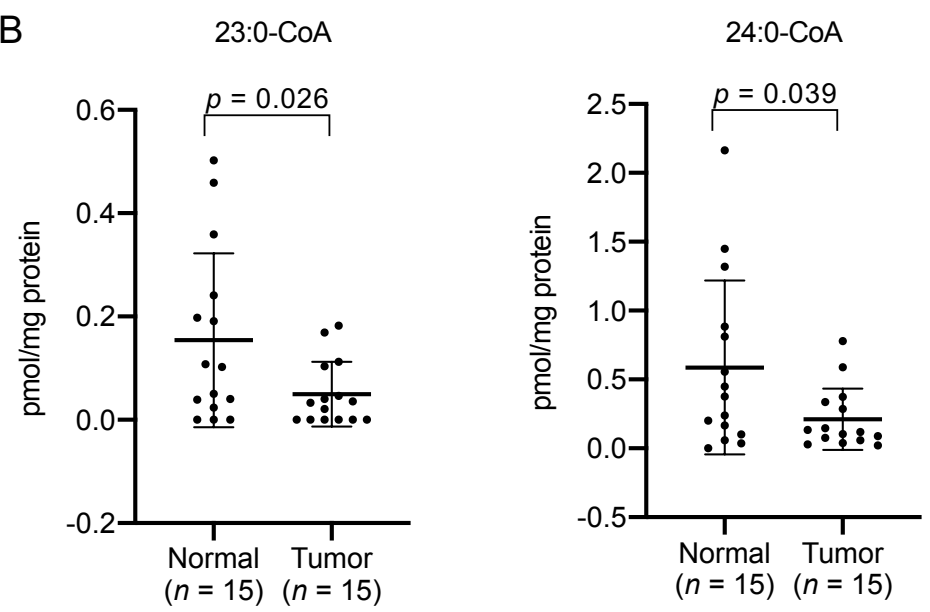

$$
\begin{aligned}
& 10 \leq \\
& 1.0<\wedge \leq 10 \\
& 0.1<\wedge \leq 1.0 \\
& <0.1 \\
& <\text { Lower limit of } \\
& \text { quantitation range }
\end{aligned}
$$

Fig. 1 The amount of acyl-CoA species in CRC tissues

(A) Each acyl-CoA species in adjunct normal tissues $(n=15)$ was quantified, and was classified according to the number of carbon and double bonds in the acyl moiety. The ratio of peak area for each acyl-CoA/D ${ }_{31}-16: 0-C o A$ was used to calculate the amount of each acyl-CoA species and the mean quantity of each acyl-CoA species in the tissues is represented with a color key. The data are also summarized in Table 1. Acyl-CoA species observed to be present below the quantitation range are indicated in gray. The total amount of the top three acyl-CoA species (16:0-, 18:0-, 18:1-CoA) comprises more than 50\% of the total amount of all acyl-CoA species, analyzed within quantitative range. (B) The amount of 23:0- and 24:0-CoA species in CRC tissues and adjunct normal tissues. The amount of 23:0- and 24:0-CoA species in CRC tissues was significantly smaller than in adjunct normal tissues. Data represent the mean \pm SD. Statistical analysis was performed with two-tailed Paired $t$-test in (B). 
A

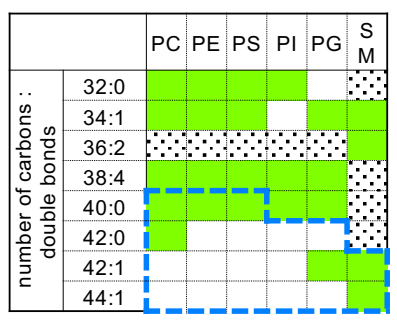

Control > Tumor $(p<0.05)$

$\because$ Not analyzed

E_- PL species with VLCFA moiety

PC 24:0/16:0

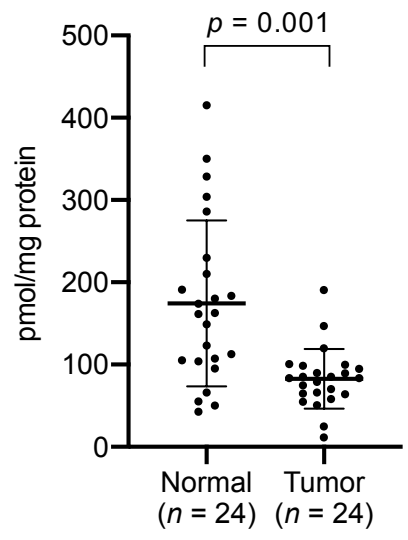

C

SM d18:1/16:0

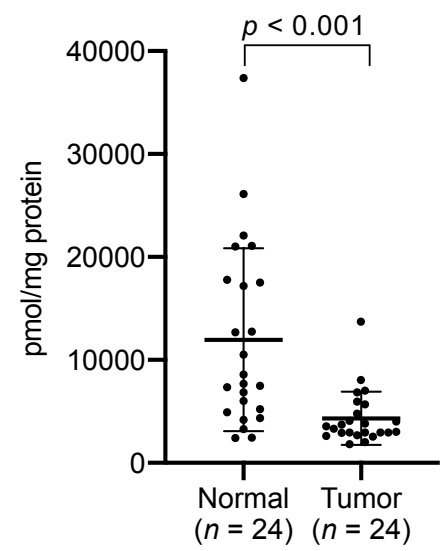

B

PC 16:0/16:0

PC 16:0/18:1

PC 18:0/20:4
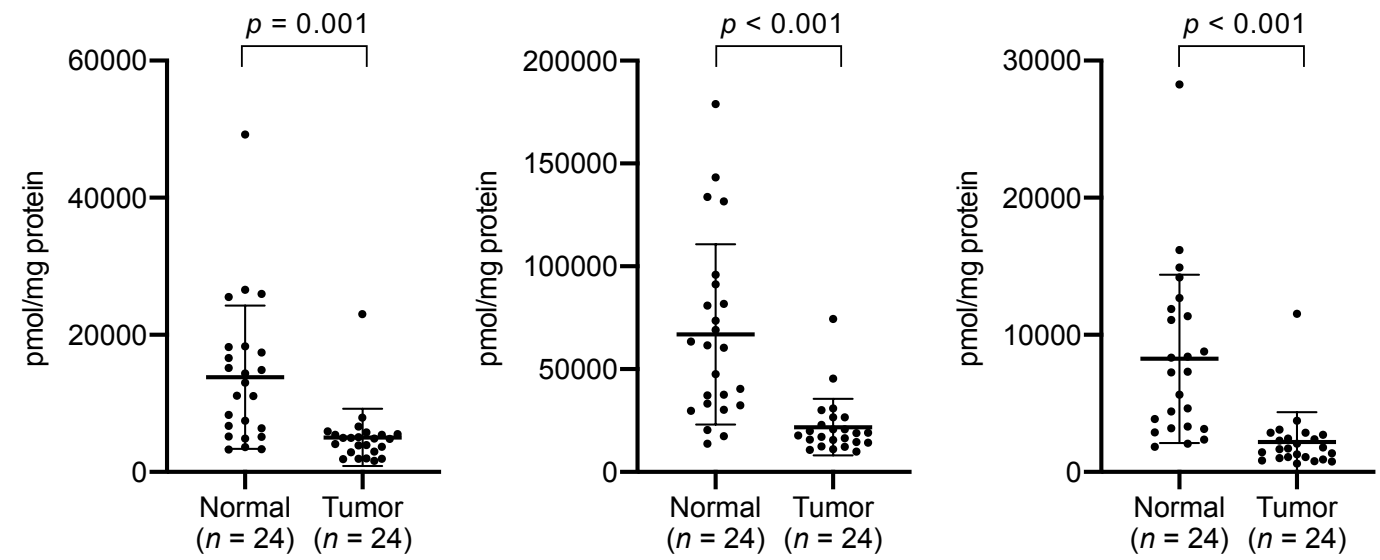

PC 26:0/16:0
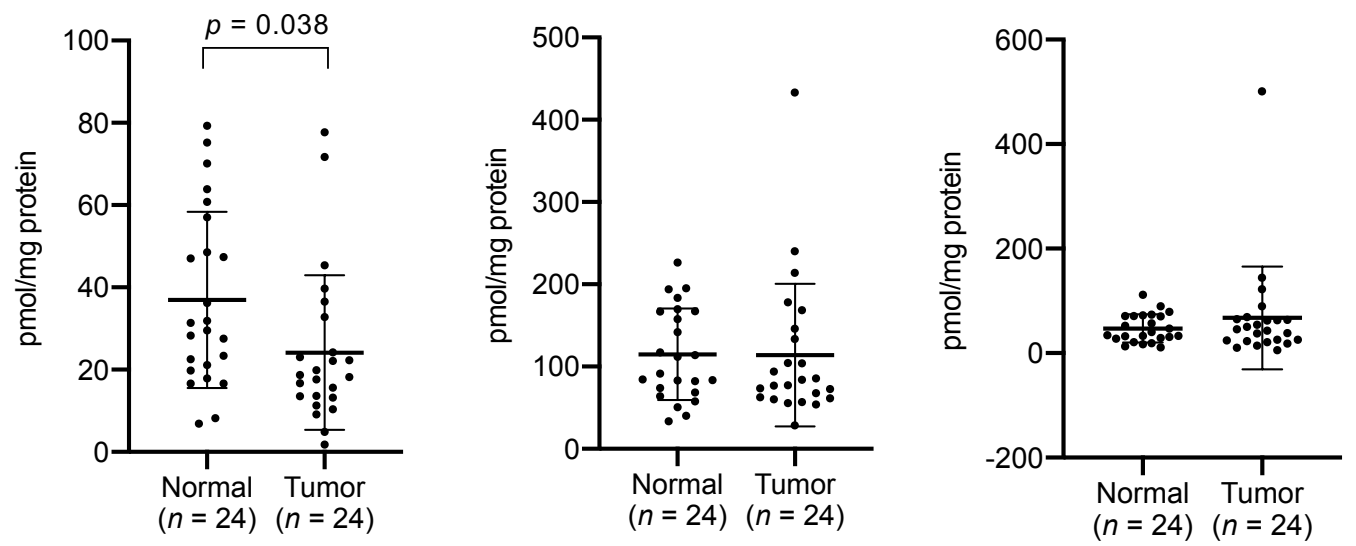

SM d18:1/24:0

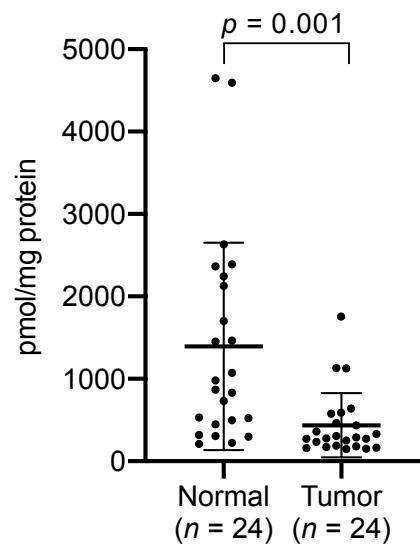

SM d20:1/24:0

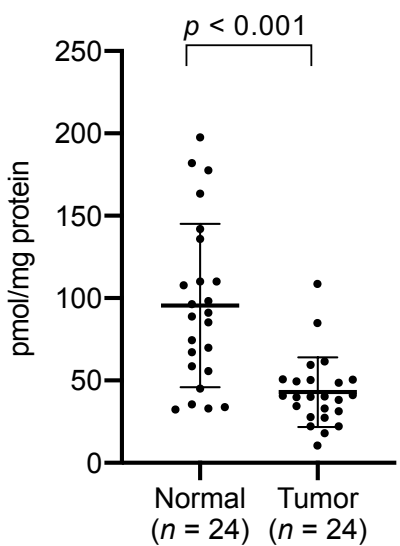

Fig. 2 Quantity of each phospholipid (PL) species in the CRC tissues

(A) The amount of each PL species was quantified by LC-MS in positive ion mode. The lipid species present in quantities significantly lower in CRC tissues $(n=24)$ relative to the adjunct normal tissues $(n=24)$ is presented in lime respectively. PL species for which the multiple reaction monitoring (MRM) channels were not designed are indicated in dot box. PL species with VLCFA moiety (C24:0 and C26:0) are indicated in blue dashed line. The amount of each phosphatidylcholine (PC) (B) and sphingomyelin (SM) species (C) are represented. The acyl-moieties for each PL species were determined by LC-MS/MS/MS in negative ion mode. 


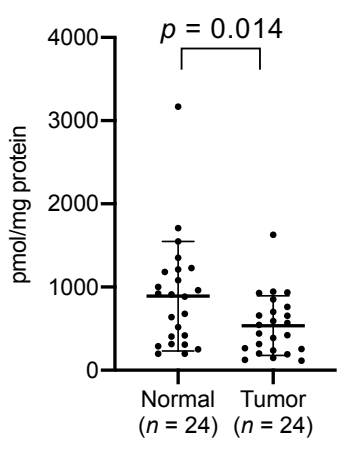

FFA 20:0

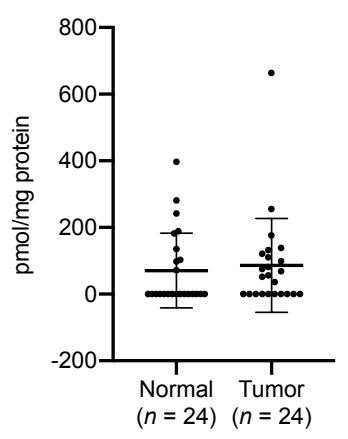

FFA 22:0

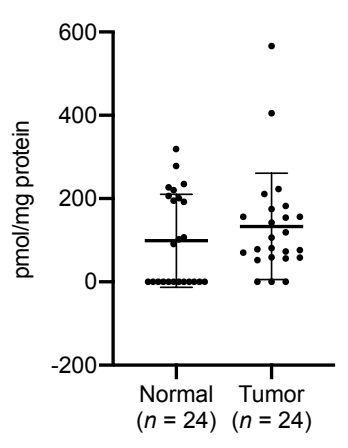

FFA 24:1

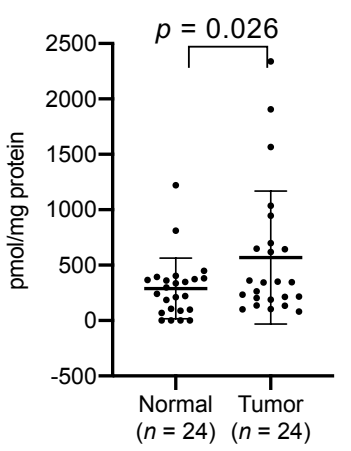

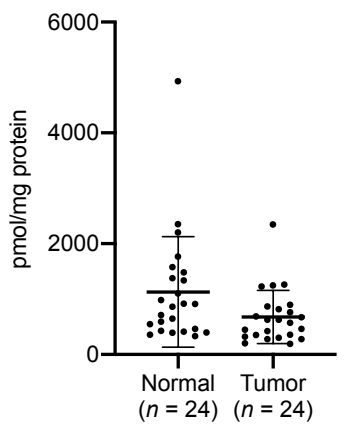

FFA 20:1

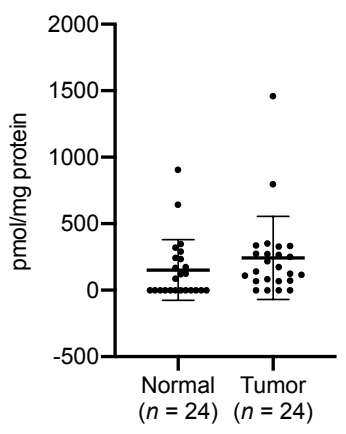

FFA 22:1

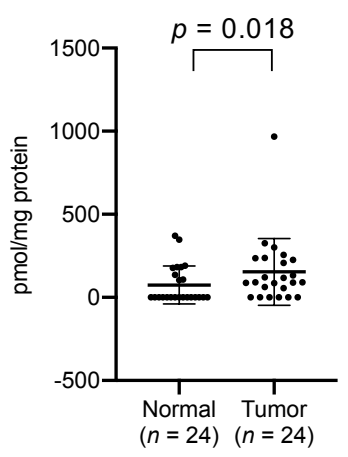

FFA 26:0

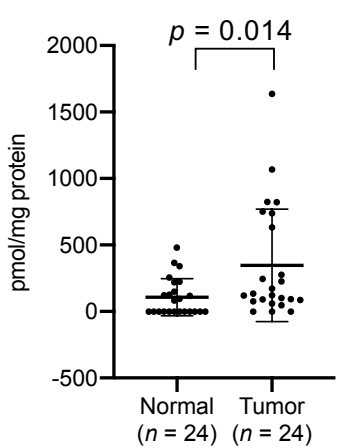

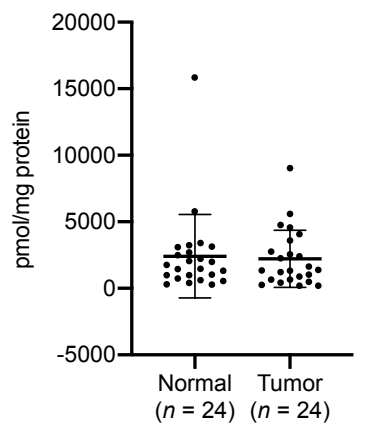

FFA 20:4

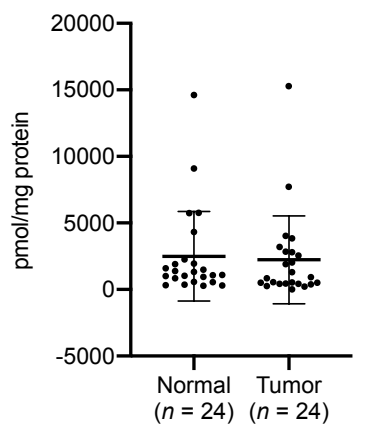

FFA 22:6

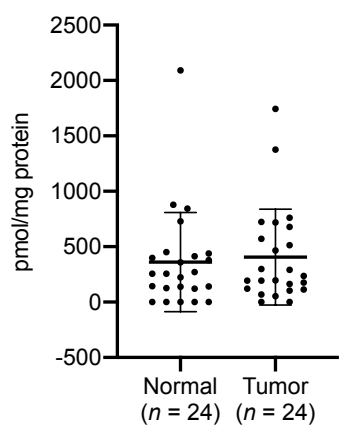

FFA 26:1

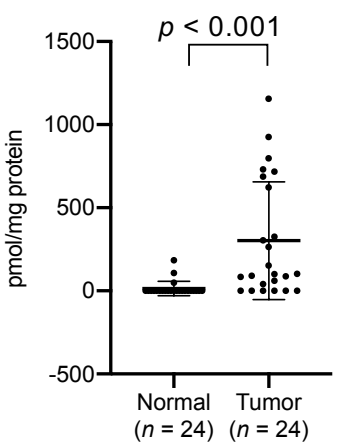

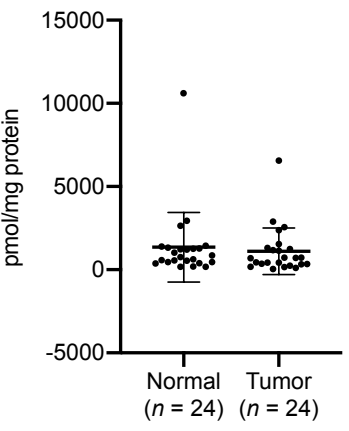

FFA 20:5

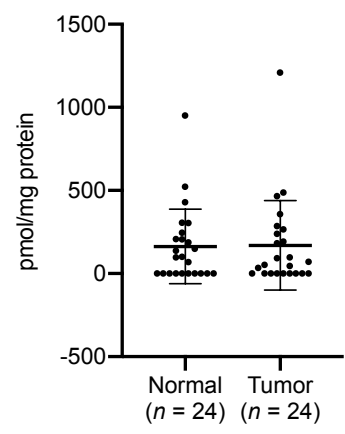

FFA 24:0

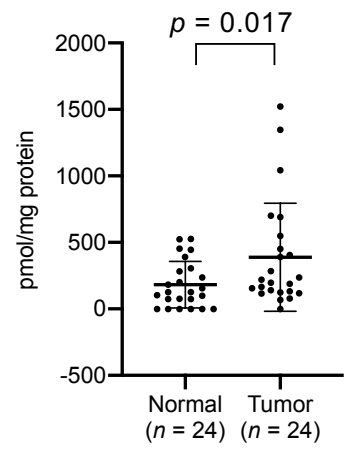

B

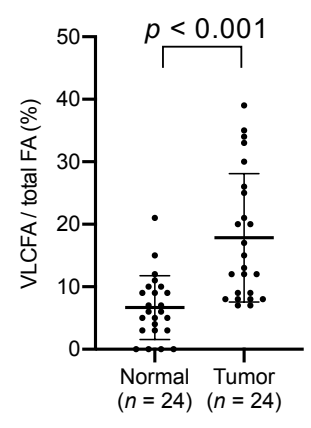

Fig. 3 Quantity of each non-esterified fatty acid (free fatty acid, FFA) species in the colorectal cancer (CRC) tissues Each FFA species (A) and the portion of non-esterified VLCFA (B) was analyzed by LC-MS. All of the non-esterified VLCFA consisting of FFA 24:0, 24:1, 26:0 and 26:1 were accumulated in CRC tissues. Data represent the mean \pm SD. Statistical analysis was performed with two-tailed Paired $t$-test in ( $A$ and $B$ ). 

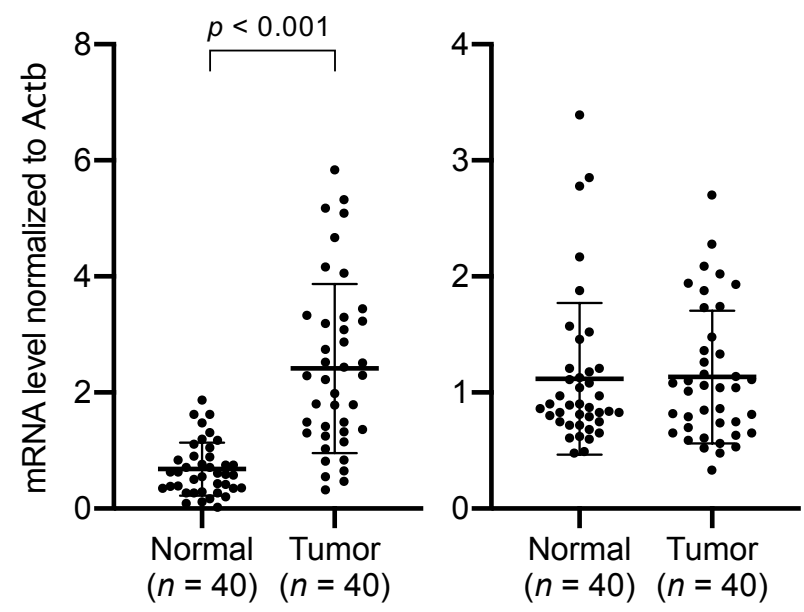

B
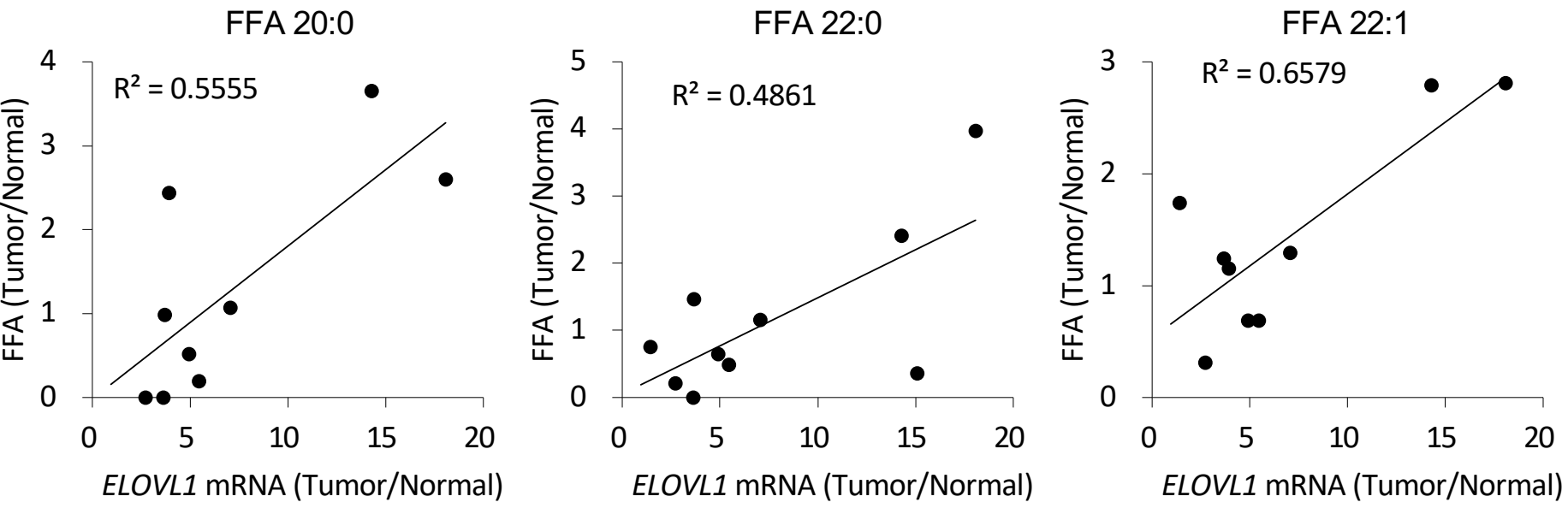

FFA 24:1

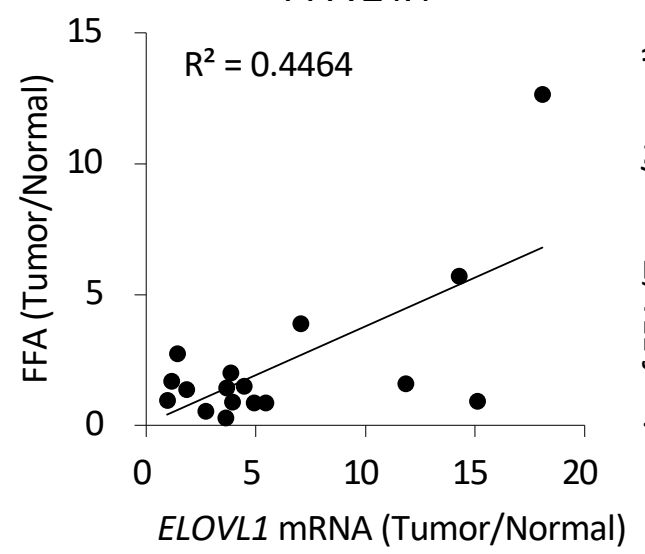

FFA 26:1

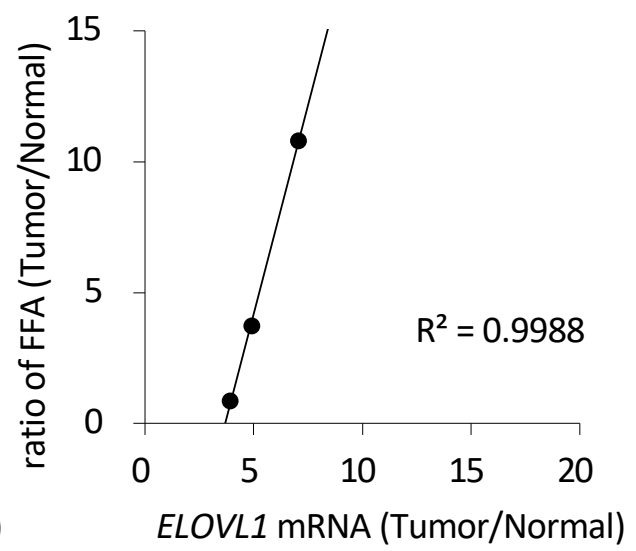

Fig. 4 The expression level of ELOVL1 is correlated with saturated and mono-unsaturated non-esterified fatty acids with no less than 20 carbons

(A) The mRNA level of ELOVL1 and ABCD1 in CRC tissues and adjunct normal tissues. The expression level of ELOVL1 was significantly higher in CRC tissues. Data represent the mean $\pm \mathrm{SD}$. (B) The correlation between the CRC/normal ratio of ELOVL1 mRNA and FFA. The higher ratio of ELOVL1 mRNA was positively correlated with the ratio of saturated and mono-unsaturated non-esterified fatty acids with no less than 20 carbons. Statistical analysis was performed with two-tailed Paired t-test in (A) and Pearson correlation coefficient in (B). 


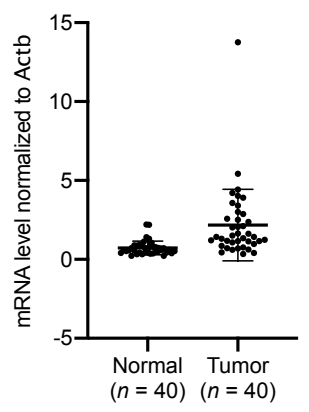

ACSL6

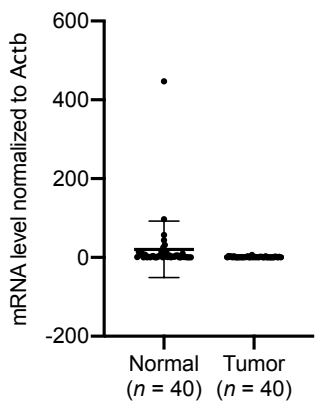

FATP4

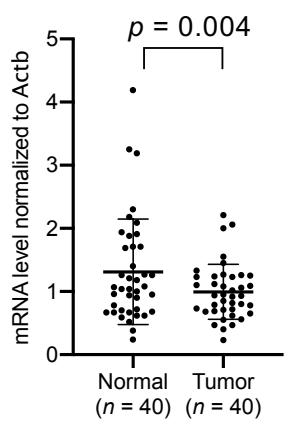

ACSBG2

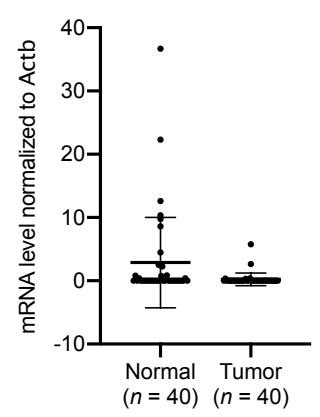

ACER2

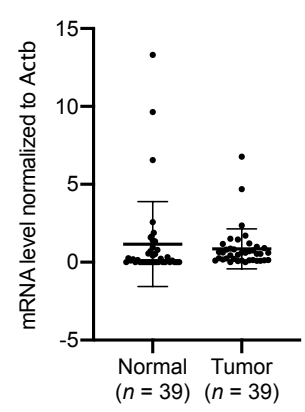

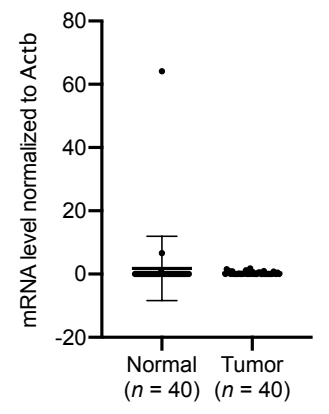

FATP1

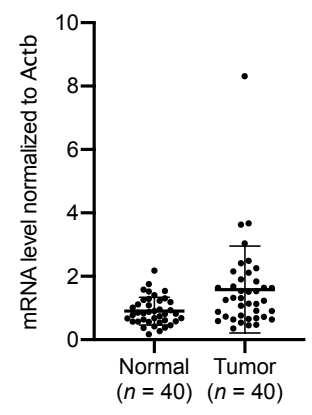

FATP5

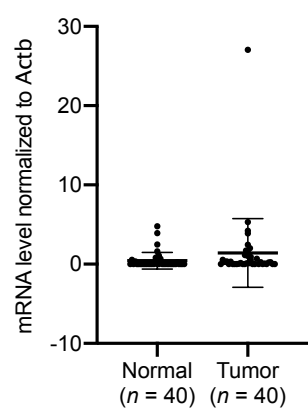

ASAH1

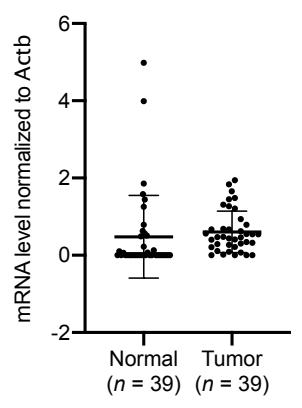

ACER3

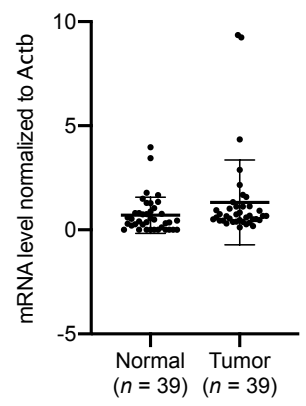

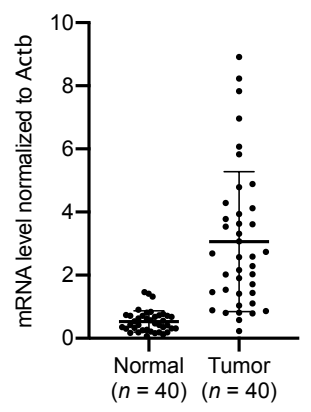

FATP2

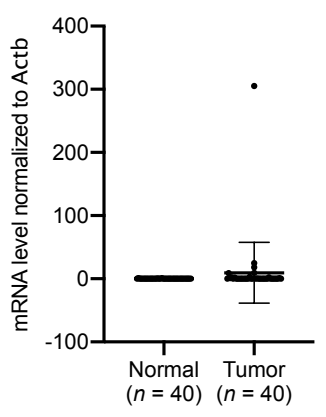

FATP6

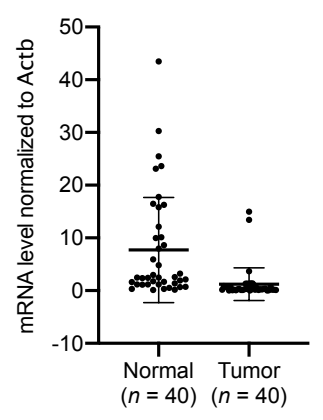

ASAH2

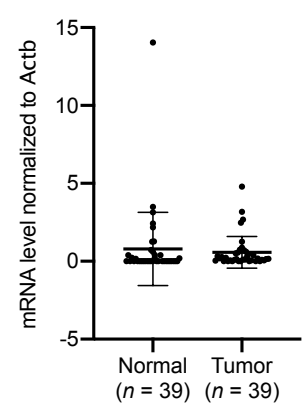

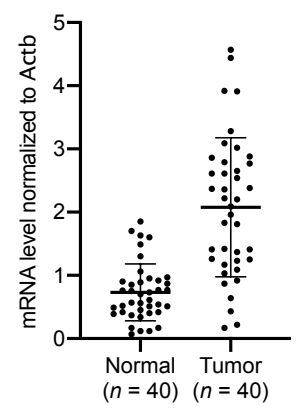

FATP3

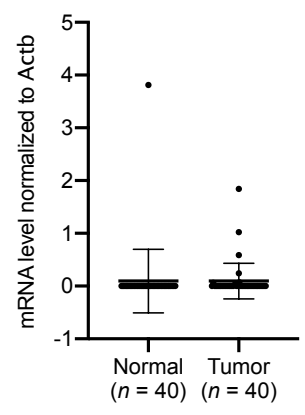

ACSBG1

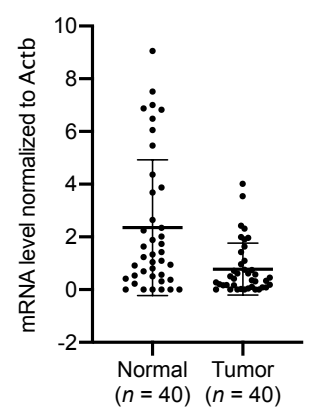

ACER1

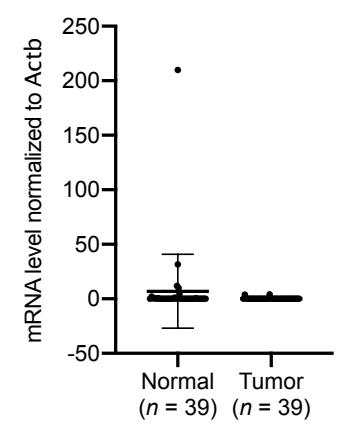

Fig. 5 The expression level of acyl-CoA synthetases and ceramidases in CRC tissues

The mRNA level of 13 acyl-CoA synthetases (ACSL1, 3-6, FATP1-6, and ACSBG1 and 2) and 5 ceramidases (ASAH1 and 2, and ACER13) in CRC and adjunct normal tissues was quantified by quantitative PCR analysis. The expression level of FATP4 was significantly lower in CRC tissues. Data represent the mean \pm SD. 


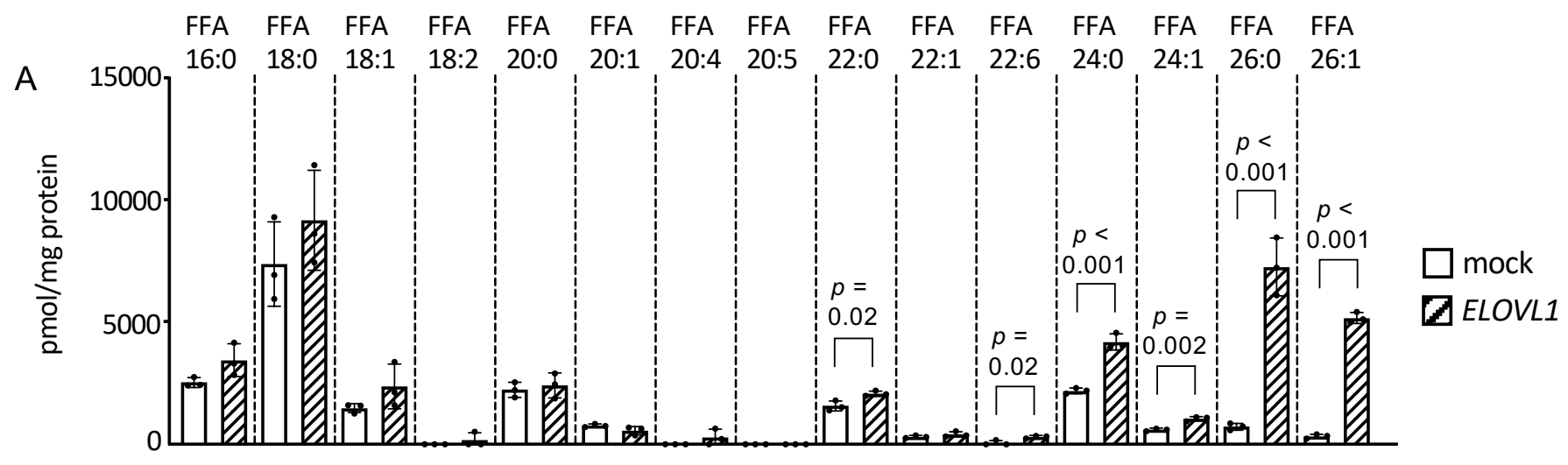

B
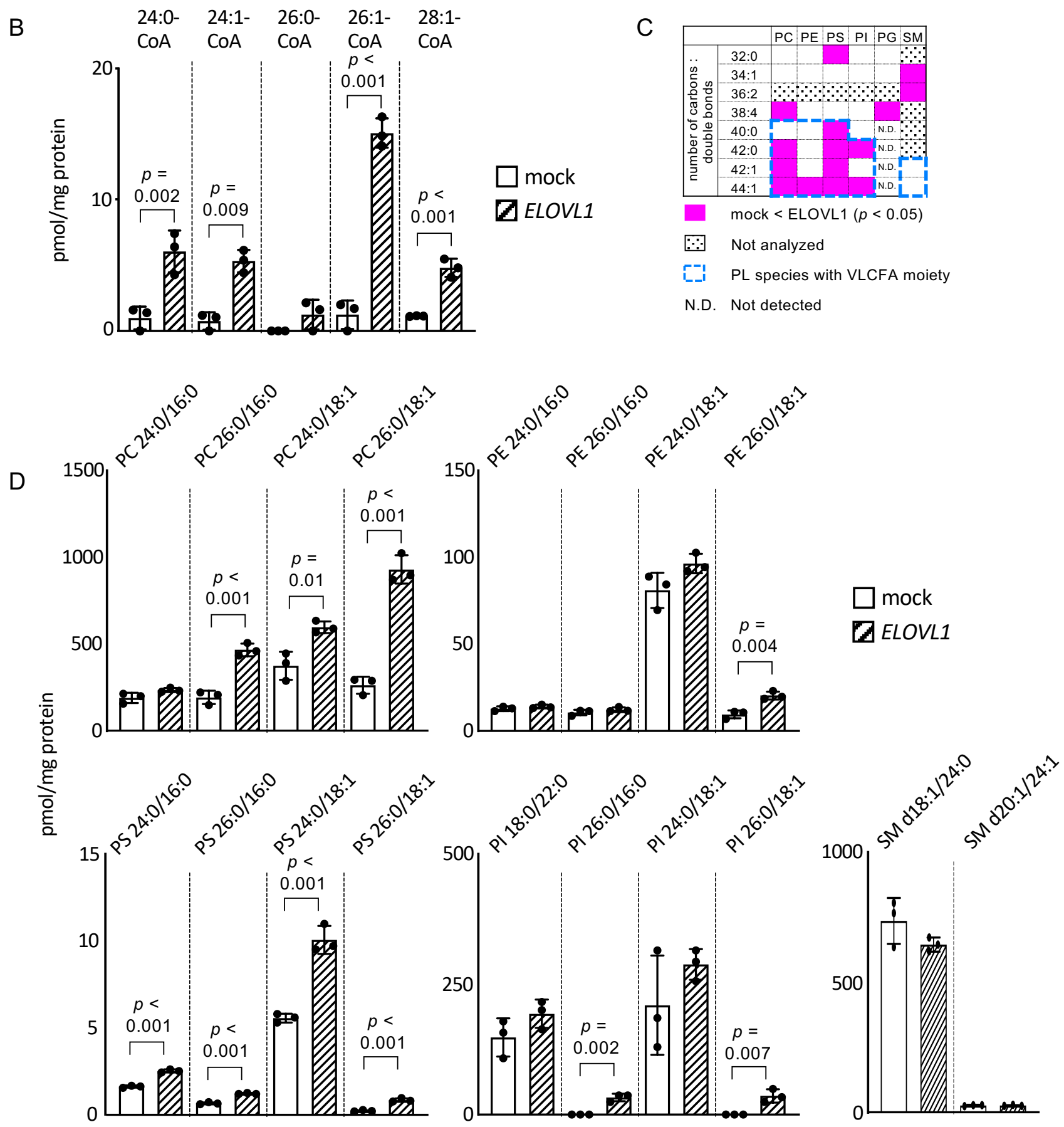

Fig. 6 
Fig. 6 The amount of FFA (A), acyl-CoA (B) and PL (C, D) in HEK293T cells transfected with pcDNA3.1-human ELOVL1. (C) The lipid species present in quantities significantly higher in ELOVL1 overexpressing cells than in mock transfected cells are presented in magenta. PL species for which the multiple reaction monitoring (MRM) channels were not designed are indicated in dot box. PL species with VLCFA moiety (C24:0 and C26:0) are indicated in blue dashed line. (D) The amount of each PL species with VLCFA moiety are represented. The acylmoieties for each PL species were determined by LC-MS/MS/MS in negative ion mode. Significant changes were not observed in the amount of PG species with VLCFA moiety. Note that both non-esterified (FFA) and esterified VLCFA (acyl-COA and PL) were significantly accumulated in HEK293T cells transfected with ELOVL1. Data represent the mean \pm SD. Statistical analysis was performed with Student's two-tailed $t$-test in (A-D). 
A

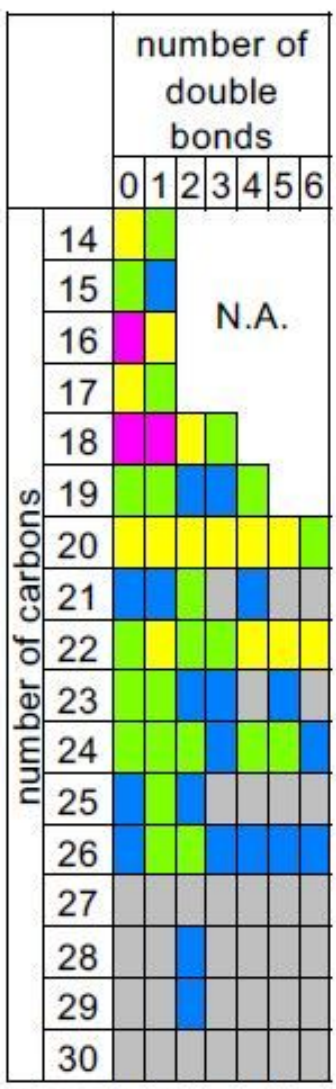

(pmol/mg protein)

$10 \leq$

$1.0<\wedge \leq 10$

$0.1<\wedge \leq 1.0$

$<0.1$

$<$ Lower limit of quantitation range
B

23:0-CoA

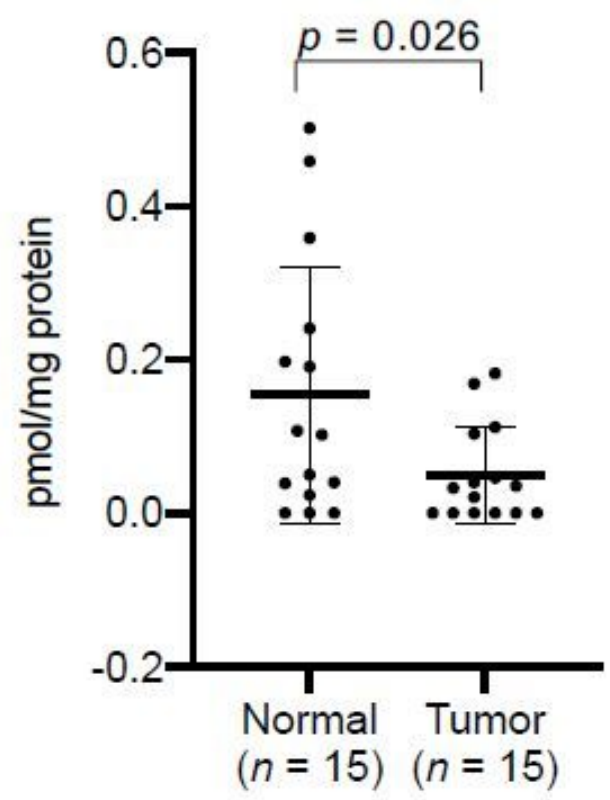

24:0-CoA

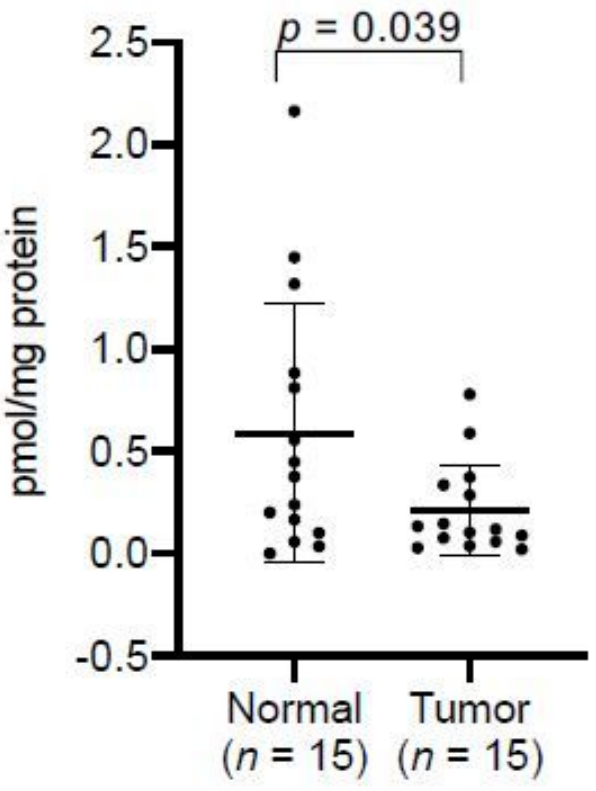

\section{Figure 1}

The amount of acyl-CoA species in CRC tissues (A) Each acyl-CoA species in adjunct normal tissues ( $\mathrm{n}=$ 15) was quantified, and was classified according to the number of carbon and double bonds in the acyl moiety. The ratio of peak area for each acyl-CoA/D31-16:0-CoA was used to calculate the amount of each acyl-CoA species and the mean quantity of each acyl-CoA species in the tissues is represented with a color key. The data are also summarized in Table 1. Acyl-CoA species observed to be present below the quantitation range are indicated in gray. The total amount of the top three acyl-CoA species (16:0-, 18:0-, 18:1-CoA) comprises more than $50 \%$ of the total amount of all acyl-CoA species, analyzed within 
quantitative range. (B) The amount of 23:0- and 24:0-CoA species in CRC tissues and adjunct normal tissues. The amount of 23:0- and 24:0-CoA species in CRC tissues was significantly smaller than in adjunct normal tissues. Data represent the mean $\pm S D$. Statistical analysis was performed with two-tailed Paired t-test in (B).

A

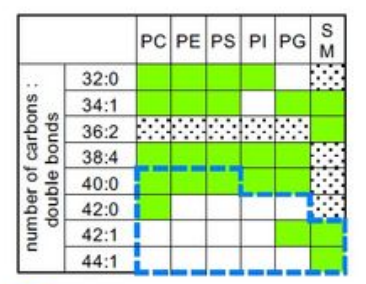

Control $>$ Tumor $(p<0.05)$

Not analyzed

[-] PL species with VLCFA moiety

B

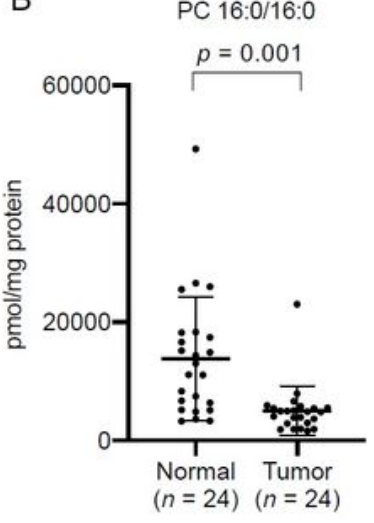

PC 24:0/16:0

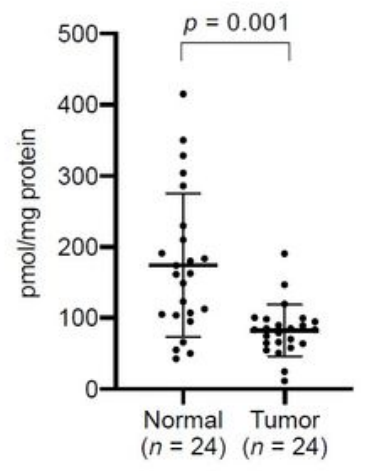

C

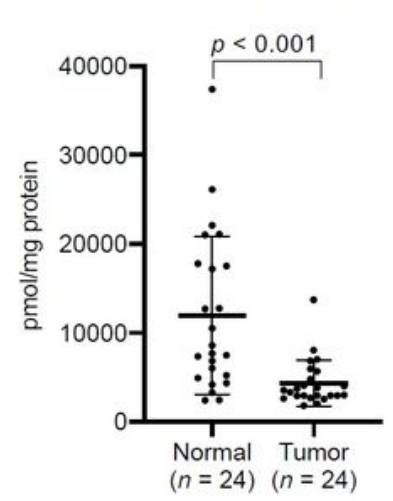

PC 26:0/16:0
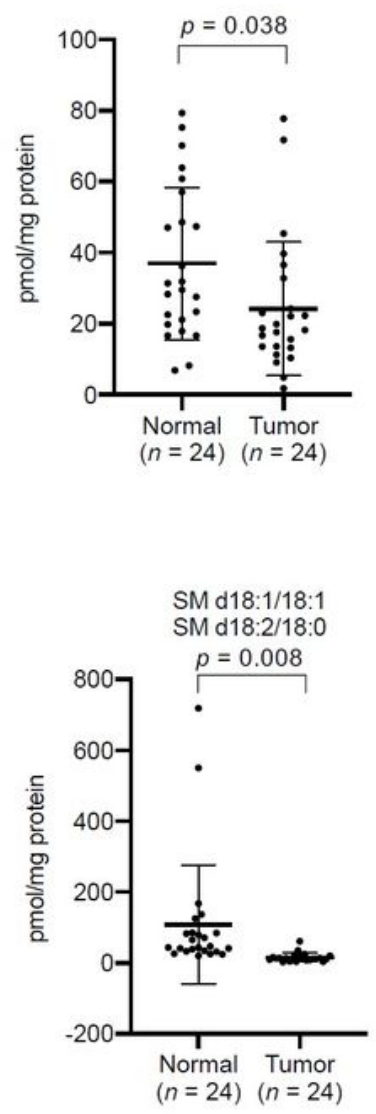

PC 16:0/18:1

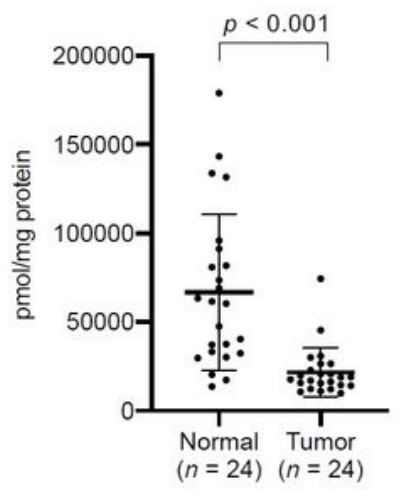

PC 24:0/18:1
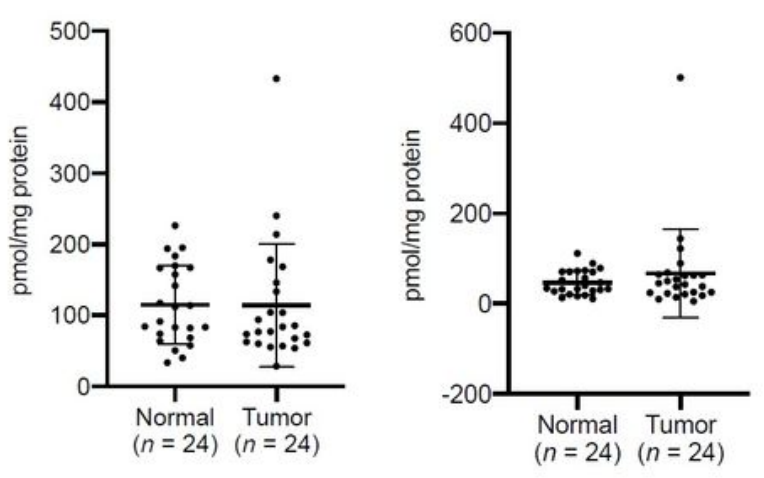

SM d 18:1/24:0

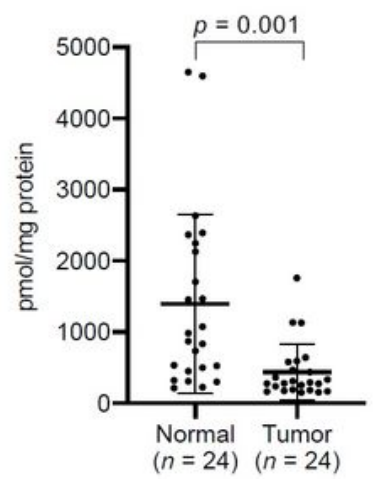

PC 18:0/20:4

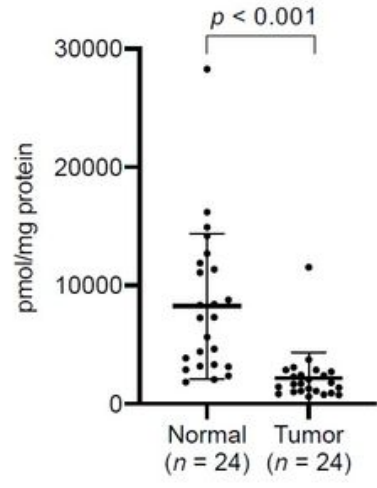

PC 26:0/18:1 
Quantity of each phospholipid (PL) species in the CRC tissues (A) The amount of each PL species was quantified by LC-MS in positive ion mode. The lipid species present in quantities significantly lower in CRC tissues $(n=24)$ relative to the adjunct normal tissues $(n=24)$ is presented in lime respectively. PL species for which the multiple reaction monitoring (MRM) channels were not designed are indicated in dot box. PL species with VLCFA moiety (C24:0 and C26:0) are indicated in blue dashed line. The amount of each phosphatidylcholine (PC) (B) and sphingomyelin (SM) species (C) are represented. The acylmoieties for each PL species were determined by LC-MS/MS/MS in negative ion mode. Data represent the mean $\pm S D$. Statistical analysis was performed with two-tailed Paired t-test in (A and B). 
A

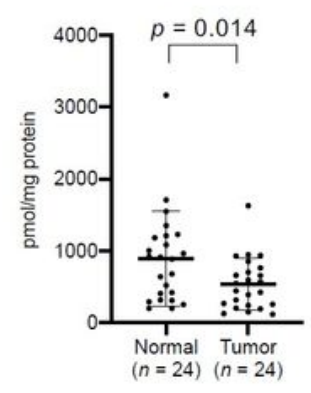

FFA 20:0

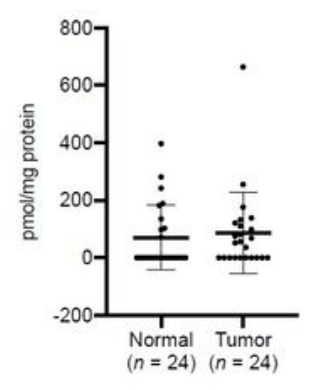

FFA 22:0

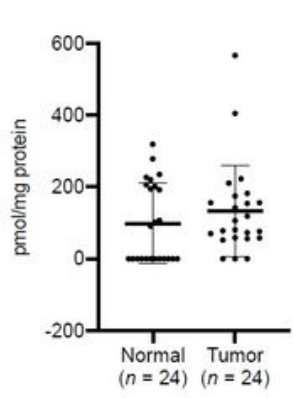

FFA 24:1

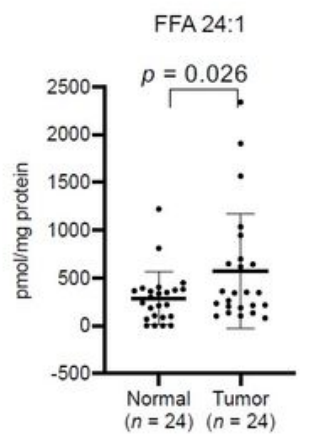

FFA 18:0

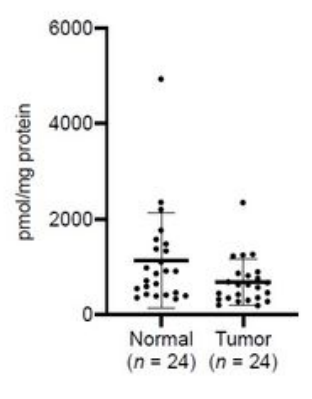

FFA 20:1

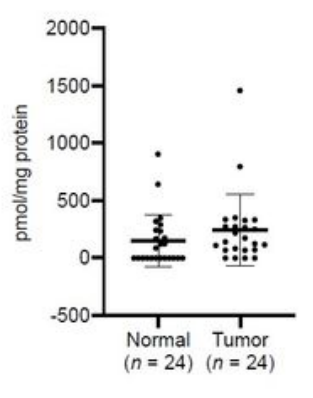

FFA 22:1

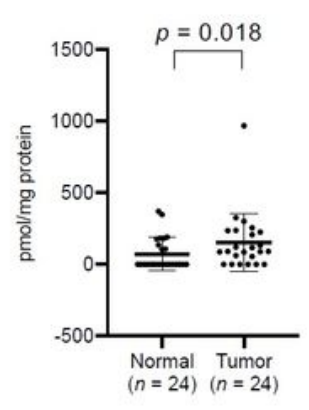

FFA 26:0

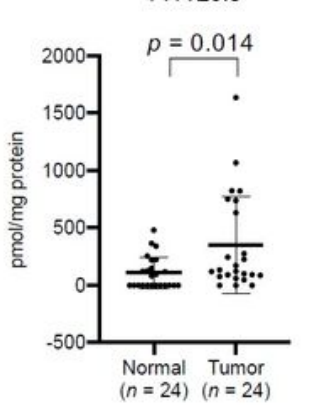

FFA $18: 1$
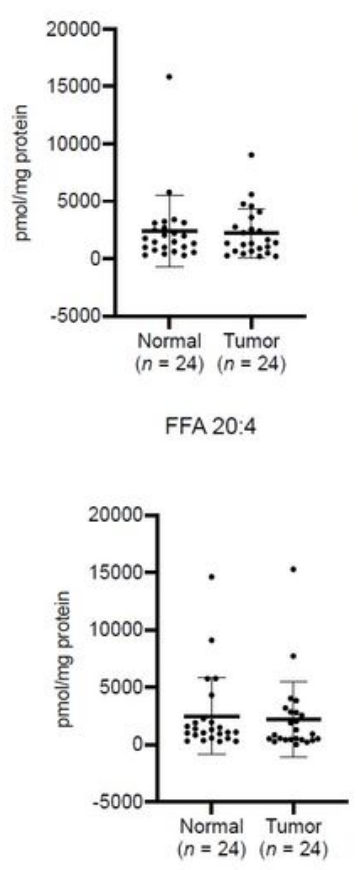

FFA 22:6
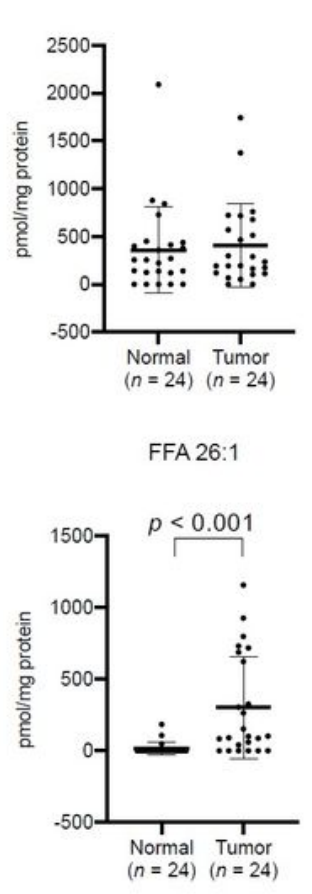

FFA 18:2
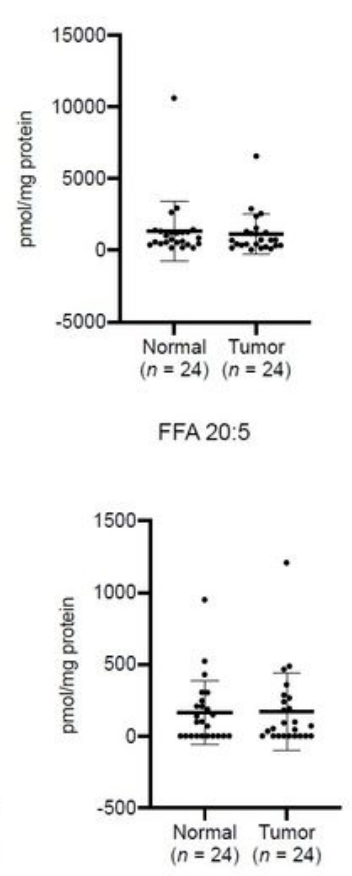

FFA 24:0

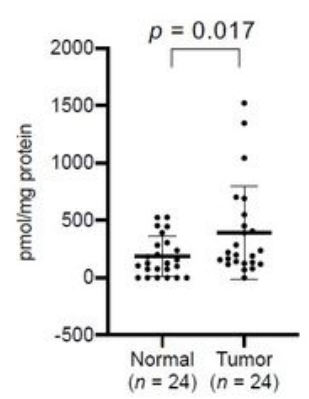

B

Ratio of VLCFA

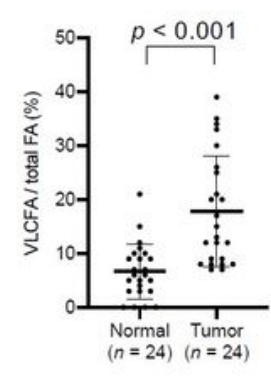

\section{Figure 3}

Quantity of each non-esterified fatty acid (free fatty acid, FFA) species in the colorectal cancer (CRC) tissues Each FFA species (A) and the portion of non-esterified VLCFA (B) was analyzed by LC-MS. All of the non-esterified VLCFA consisting of FFA 24:0, 24:1, 26:0 and 26:1 were accumulated in CRC tissues. Data represent the mean \pm SD. Statistical analysis was performed with two-tailed Paired t-test in ( $A$ and B). 


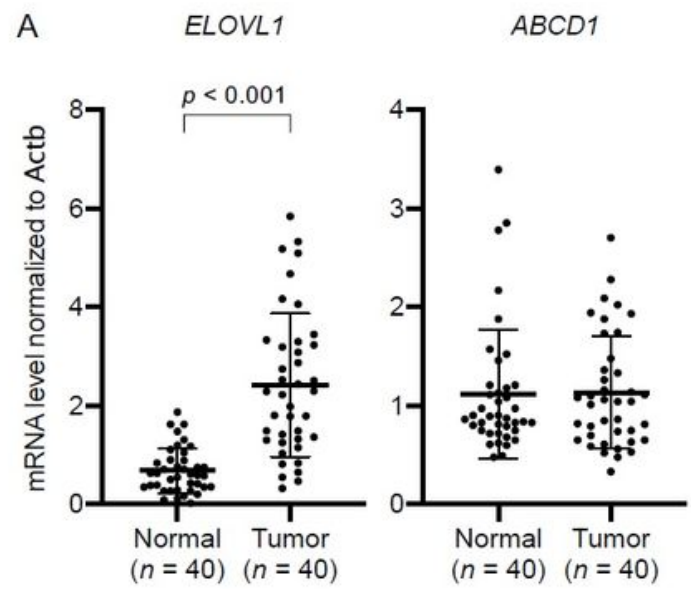

B

FFA 20:0

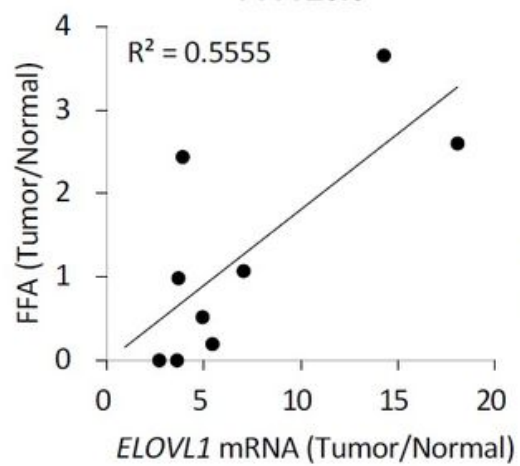

FFA 22:0

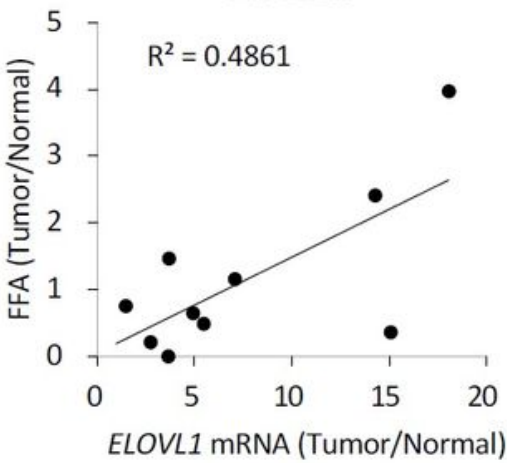

FFA 22:1

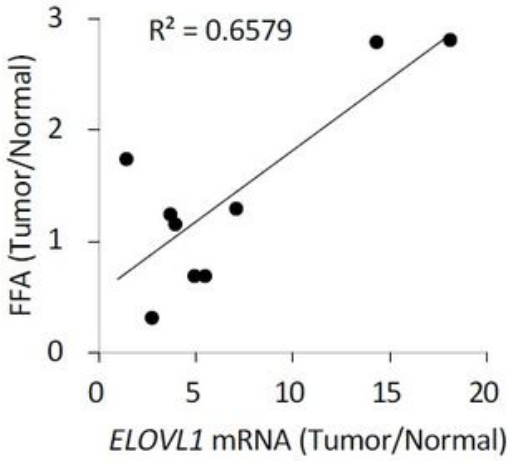

FFA 24:1

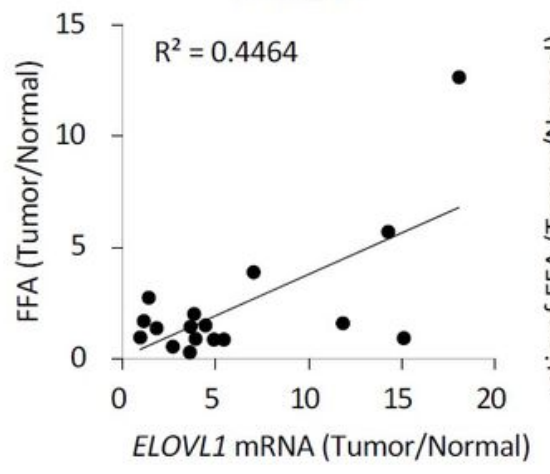

FFA 26:1

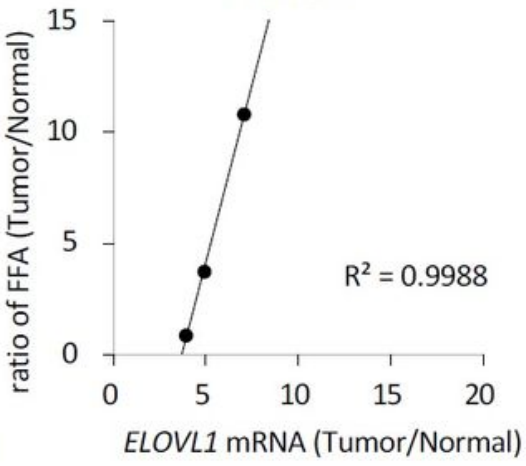

Figure 4

The expression level of ELOVL1 is correlated with saturated and mono-unsaturated non-esterified fatty acids with no less than 20 carbons (A) The mRNA level of ELOVL1 and ABCD1 in CRC tissues and adjunct normal tissues. The expression level of ELOVL1 was significantly higher in CRC tissues. Data represent the mean $\pm S D$. (B) The correlation between the CRC/normal ratio of ELOVL1 mRNA and FFA. The higher ratio of ELOVL1 mRNA was positively correlated with the ratio of saturated and mono- 
unsaturated non-esterified fatty acids with no less than 20 carbons. Statistical analysis was performed with two-tailed Paired t-test in (A) and Pearson correlation coefficient in (B).
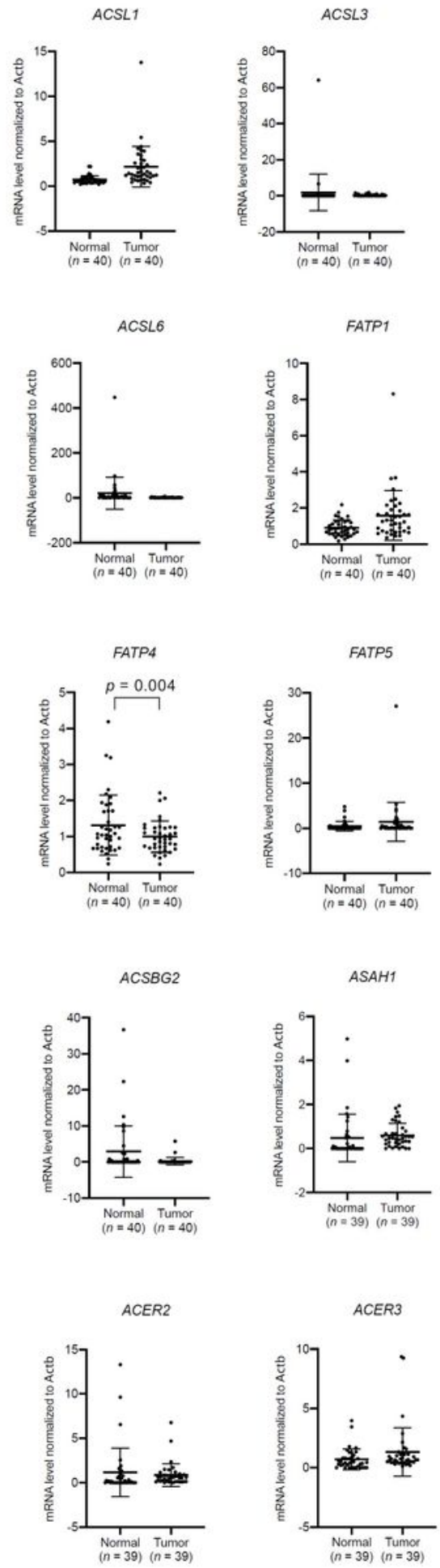
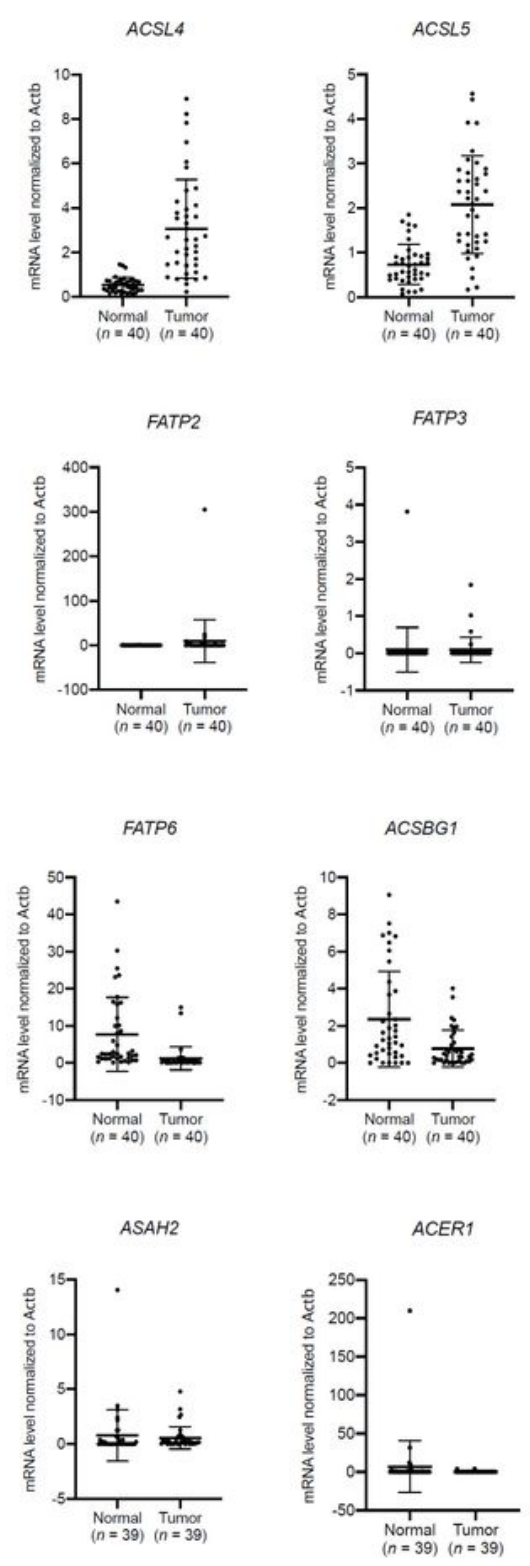

\section{Figure 5}

The expression level of acyl-CoA synthetases and ceramidases in CRC tissues The mRNA level of 13 acylCoA synthetases (ACSL1, 3-6, FATP1-6, and ACSBG1 and 2) and 5 ceramidases (ASAH1 and 2, and ACER1-3) in CRC and adjunct normal tissues was quantified by quantitative PCR analysis. The 
expression level of FATP4 was significantly lower in CRC tissues. Data represent the mean $\pm S D$. Statistical analysis was performed with two-tailed Paired t-test.
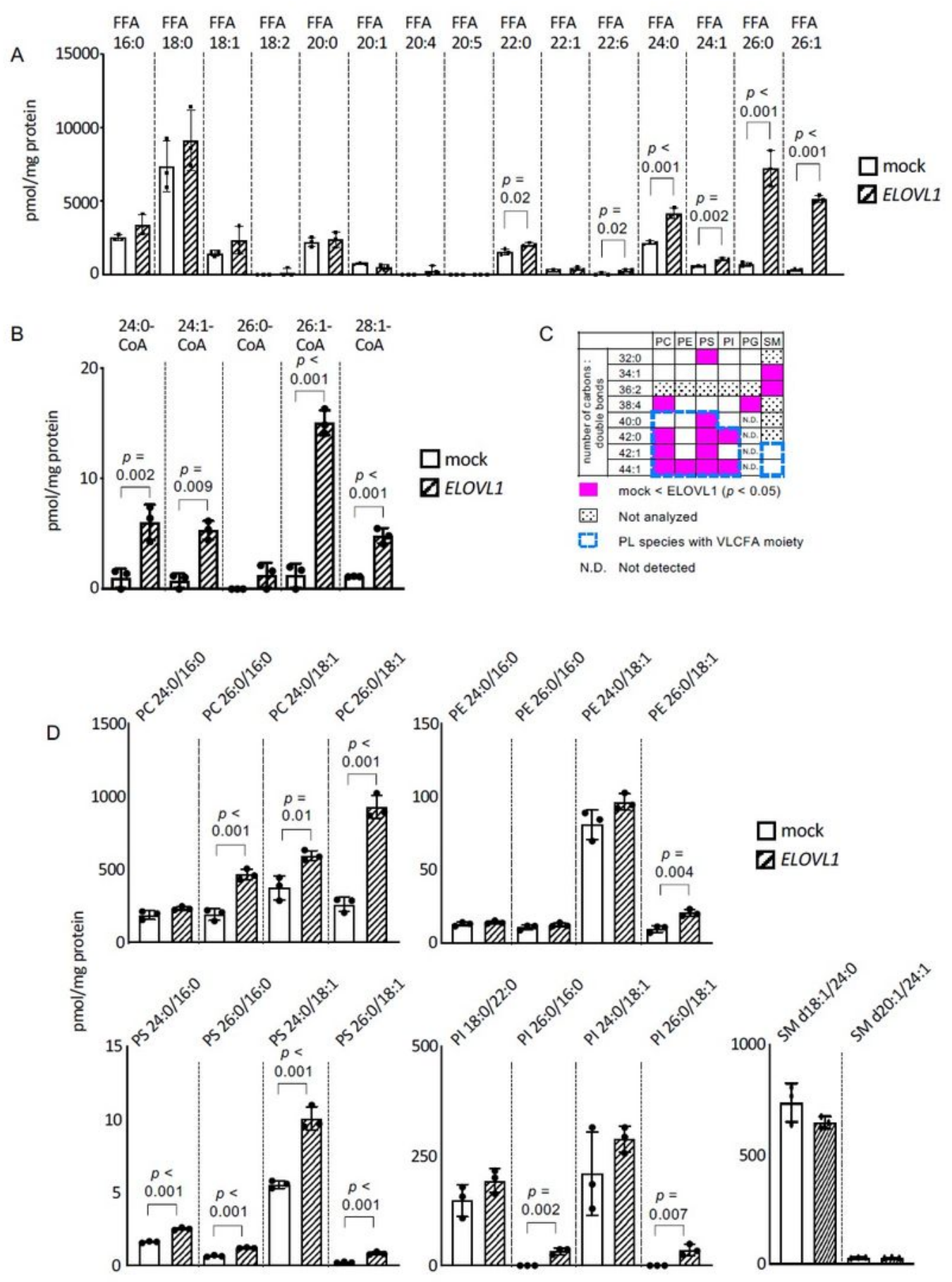

Figure 6

The amount of FFA (A), acyl-CoA (B) and PL (C, D) in HEK293T cells transfected with pcDNA3.1-human ELOVL1. (C) The lipid species present in quantities significantly higher in ELOVL1 overexpressing cells than in mock transfected cells are presented in magenta. PL species for which the multiple reaction 
monitoring (MRM) channels were not designed are indicated in dot box. PL species with VLCFA moiety (C24:0 and C26:0) are indicated in blue dashed line. (D) The amount of each PL species with VLCFA moiety are represented. The acylmoieties for each PL species were determined by LC-MS/MS/MS in negative ion mode. Significant changes were not observed in the amount of PG species with VLCFA moiety. Note that both non-esterified (FFA) and esterified VLCFA (acyl-CoA and PL) were significantly accumulated in HEK293T cells transfected with ELOVL1. Data represent the mean \pm SD. Statistical analysis was performed with Student's two-tailed t-test in (A-D).

\section{Supplementary Files}

This is a list of supplementary files associated with this preprint. Click to download.

- 3SciRepCRCSupplemental201118.pdf 